Ann. Scient. Éc. Norm. Sup.,

$4^{\mathrm{e}}$ série, t. 40, 2007, p. 845 à 884.

\title{
INTEGRABILITY OF HAMILTONIAN SYSTEMS AND DIFFERENTIAL GALOIS GROUPS OF HIGHER VARIATIONAL EQUATIONS
}

\author{
BY JUAN J. MORALES-RUIZ，JEAN-PIERRE RAMIS AND \\ CARLES SIMÓ
}

\begin{abstract}
Given a complex analytical Hamiltonian system, we prove that a necessary condition for its meromorphic complete integrability is the commutativity of the identity component of the Galois group of each variational equation of arbitrary order along any integral curve. This was conjectured by the first author based on a suggestion by the third author. The first-order non-integrability criterion, obtained by the first and second authors using only first variational equations, is extended to higher orders by the present criterion. Using this result (at order two, three or higher) it is possible to solve important open problems of integrability which escaped the first order criterion.
\end{abstract}

(c) 2008 Elsevier Masson SAS

RÉSUMÉ. - Nous montrons qu'étant donné un système hamiltonien analytique complexe, une condition nécessaire pour qu'il soit méromorphiquement intégrable, au sens de Liouville, est que la composante connexe de l'identité du groupe de Galois différentiel de toute équation variationnelle, d'ordre arbitraire, le long de toute courbe intégrale, soit commutative. Ceci avait été conjecturé par le premier auteur, à la suite d'une suggestion du troisième, motivée par des observations numériques et analytiques sur des équations variationnelles d'ordre supérieur. Le critère présenté dans cet article étend aux équations d'ordre supérieur le critère de Morales-Ramis, obtenu antérieurement par les deux premiers auteurs, qui n'utilisait que la première équation variationnelle. Utilisant le nouveau critère (aux ordres deux, trois ou plus), il est possible de résoudre d'importants problèmes d'intégrabilité pour lesquels le critère de Morales-Ramis ne permettait pas de conclure.

(c) 2008 Elsevier Masson SAS

\section{Introduction}

The problem of integrability by quadratures of dynamical systems is a particularly old, important and difficult one. We know that, given an algebraic or analytic dynamical system, defined by ordinary differential equations, a solution always exists locally and can be sometimes prolonged for arbitrary time values, whether negative or positive. This said, and much in the spirit of the eighteenth-century mathematician, an attempt could be made at finding the general solution analytically in an "explicit" way. Whenever this is possible, we call the system "integrable". Unfortunately, it is an empirical fact that a "general" dynamical system does not adhere to any known definition of integrability, and that, moreover, such a unified definition is nowadays unavailable for arbitrary dynamical systems. In other words, the "majority" of dynamical systems are non-integrable (one can even suspect integrability is a codimension infinity property in some 
reasonable sense) and it is impossible to find their general solution in closed form. For some remarks about the meaning of integrability see [60]. The situation is similar to the problem of solvability by radicals of algebraic equations, and the fact that our approach in this paper follows a Galoisian path should not come across as surprising.

In this paper we will only consider analytical dynamical systems over the complex field; in the applications it is necessary to go back to the real field, a task which is not always easy to perform. This said, there are at least two families of finite-dimensional complex-analytical dynamical systems for which the notion of integrability is well-defined: Hamiltonian systems and linear differential equations. For Hamiltonian systems, integrability is defined in the sense of Liouville: the existence of a complete set of independent first integrals in pairwise involution. Whenever this happens the Hamiltonian system is said to be completely integrable, or simply integrable for the sake of simplicity. For linear ordinary differential equations, integrability is defined in the context of differential Galois theory, also called Picard-Vessiot theory.

Let us specify both definitions a bit further. A system with Hamiltonian $H$ defined over a symplectic analytic complex manifold $M$ of (complex) dimension $2 n$,

$$
\dot{x}=X_{H}(x),
$$

is integrable if there exist $n$ first integrals $H=f_{1}, f_{2}, \ldots, f_{n}$ independent and in pairwise involution, $\left\{f_{i}, f_{j}\right\}=0,1 \leqslant i, j \leqslant n,\{$,$\} being the Poisson bracket defined by the symplectic$ form.

In general, we will assume $f_{1}, f_{2}, \ldots, f_{n}$ meromorphic, but we will sometimes require them to be somehow more regular, for instance meromorphic at $\infty$, i.e., rational functions, if $M$ is an open set (in the algebraic sense) of a complex projective space. For specific facts about the integrability of complex Hamiltonians see [59, Chapter 3].

We assume that the reader is acquainted with both the algebraic approach and the geometric connection approach to Picard-Vessiot theory. For the necessary definitions and results one can look at [59, Chapter 2]; for a more complete study, including detailed proofs and other references, a standard monograph is [80].

Given a system of linear ordinary differential equations

$$
\dot{\xi}=A \xi,
$$

with coefficients in a differential field $K, A \in \operatorname{Mat}(m, K)$, we call it integrable if its general solution is obtained by a combination of quadratures, exponentials of quadratures and algebraic functions. In other words, if $L:=K\left(u_{i j}\right)$ is the Picard-Vessiot extension of $K, u_{i j}$ being a fundamental matrix of solutions of (2), then there exists a chain of differential extensions $K_{1}:=K \subset K_{2} \subset \cdots \subset K_{r}:=L$, where each extension is given by the adjunction of one element $a, K_{i} \subset K_{i+1}=K_{i}\left(a, a^{\prime}, a^{\prime \prime}, \ldots\right)$, such that $a$ satisfies one of the following conditions:

(i) $a^{\prime} \in K_{i}$,

(ii) $a^{\prime}=b a, b \in K_{i}$,

(iii) $a$ is algebraic over $K_{i}$;

the usual terminology is that the Picard-Vessiot extension $L / K$ is Liouvillian. Then, it can be proved that a linear differential equation is integrable if, and only if, the identity component $G^{0}$ of the Galois group G of (2) (which is an algebraic group over the constant field) is solvable. In particular, if the identity component is commutative, then the equation is integrable. In this paper we will only consider the case in which the coefficient field $K$ is the field of meromorphic functions over some suitable Riemann surface.

Given a complex analytic Hamiltonian system (1) as before, we can consider a particular solution, $\phi\left(x_{0}, t\right),(x, t) \mapsto \phi(x, t)$ being the flow of equation (1). If not an equilibrium point,

$4^{\text {e }}$ SÉRIE - TOME $40-2007-\mathrm{N}^{\circ} 6$ 
$\phi\left(x_{0}, t\right)$ defines a Riemann surface $\Gamma$ immersed in $M$. The first-order variational equation $V E_{1}$ of (1) along $\Gamma$ is given by

$$
\frac{d}{d t} \frac{\partial \phi}{\partial x}\left(x_{0}, t\right)=\frac{\partial X_{H}}{\partial x}\left(\phi\left(x_{0}, t\right)\right) \frac{\partial \phi}{\partial x}\left(x_{0}, t\right)
$$

which, setting $\phi^{(1)}=\phi^{(1)}(t)=\frac{\partial \phi}{\partial x}\left(x_{0}, t\right)$, can be written as

$$
\dot{\phi}^{(1)}=\frac{\partial X_{H}}{\partial x}\left(\phi\left(x_{0}, t\right)\right) \phi^{(1)} .
$$

The solution of (4) yields the linear part of the flow $\phi(x, t)$ along $\Gamma$. Now we assume that we can complete the Riemann surface $\Gamma$ to a Riemann surface $\bar{\Gamma}$ by possibly adding equilibrium points, singularities of the Hamiltonian field $X_{H}$ and points at $\infty$, the coefficients of (4) being meromorphic at all of them. Then the differential field of coefficients of the linear differential equation (4) is by definition the field of meromorphic functions over $\bar{\Gamma}$, see [62] (or [59]) for the details. Then the first and second authors proved the following

THEOREM 1 ([62]). - If the Hamiltonian system (1) is completely integrable with meromorphic first integrals in a neighbourhood of $\Gamma$, not necessarily independent on $\Gamma$ itself, then the identity component $G^{0}$ of the Galois group $G$ of Equation (4) is commutative.

This result is a typical variant of several possible theorems in [62] when, instead of $\Gamma$, we consider a Riemann surface $\bar{\Gamma}$ obtained from $\Gamma$ by adding some points. It is possible to give equivalent versions of theorem 1 :

... then the Lie algebra of the Galois group of Equation (4) is abelian,

or

...then the Galois group $G$ of Equation (4) is virtually commutative (i.e., it admits a commutative invariant subgroup $H$ such that $G / H$ is finite).

In this last statement we can replace the differential Galois group $G$ by the monodromy group, clearly a subgroup of $G$ :

COROLlaRY 1. - If the Hamiltonian system (1) is completely integrable with meromorphic first integrals in a neighbourhood of $\Gamma$, not necessarily independent on $\Gamma$ itself, then the monodromy group of (4) is virtually commutative.

Theorem 1 follows a tradition tracing back to Poincaré, who introduced the variational equations and found a relation between integrability and the monodromy matrix along real periodic orbits [71]. More recently Ziglin considered the monodromy group of the variational equations of (1) in the complex analytic setting in order to study necessary conditions for the existence of a complete set of independent first integrals, albeit without any involution assumption [86]. For more information and precise statements about the history of the method of the variational equations in connection with the integrability problem of Hamiltonian systems, see [59]. Theorem 1 can be considered as a generalisation of the aforementioned result by Ziglin; actually, the latter is easily proved to follow from the results of [62] as a corollary (see [59]). Theorem 1, as well as its alternative versions in [62], is clearly a non-integrability criterion; since the end of the nineties it has been applied by several authors to the study of the non-integrability of a wide range of systems:

a) $N$-body problems, problems with homogeneous potentials and cosmological models [5,6, $13-15,17,16,37,38,45,46,48,49,53-55,63,64,66,69,68,78,81,84,85]$.

b) Some physical problems $[3,4,12,27-29,51,72]$. 
c) Other mechanical problems (rigid body, spring-pendulum, ... ) $[44,47,50,52,83]$.

d) Systems with some chaotic behaviour (splitting of asymptotic surfaces) $[61,82]$.

Some surveys and general expository works have been also published: [9-11,21,22].

Thus, Theorem 1 and its variants can provide for new proofs of non-integrability, in turn either solving longtime open problems or else simplifying already existing proofs, as was the case for the heavy top problem [50]. Applied to some systems, however, the sole use of this theorem is insufficient for the above purpose, even in the presence of overwhelming numerical evidence of non-integrability. This is the situation faced, for instance, for some third-order polynomial potentials-and, more generally, for some very degenerate situations in parametrised families of potentials. Considering this situation, the third author proposed the use of higher-order variational equations in order to obtain proofs of non-integrability. Then a precise conjecture was stated by the first author (see next section).

\section{Our main results}

Beyond the first-order variational equation (4), it is possible to consider the higher-order variational equations $V E_{k}$ along $\Gamma$, with $k>1$. The "fundamental" solution of $V E_{k}$ is given by $\left(\phi^{(1)}(t), \phi^{(2)}(t), \ldots, \phi^{(k)}(t)\right)$,

$$
\phi(x, t)=\phi\left(x_{0}, t\right)+\phi^{(1)}(t)\left(x-x_{0}\right)+\cdots+\phi^{(k)}(t)\left(x-x_{0}\right)^{k}+\cdots
$$

being the Taylor series up to order $k$ of the flow $\phi(x, t)$ with respect to the variable $x$ at the point $\left(x_{0}, t\right)$. That is, $\phi^{(k)}(t)=\frac{1}{k !} \frac{\partial^{k}}{\partial x_{0}^{k}} \phi\left(x_{0}, t\right)$. The initial conditions are clearly $\phi^{(1)}(0)=\mathrm{id}$ and $\phi^{(j)}(0)=0$ for all $j>1$. It must be stressed that, as opposed to alternative definitions, we regard the order $k$ variational equation as the differential system satisfied by $\left(\phi^{(1)}, \phi^{(2)}, \ldots, \phi^{(k)}\right)$ rather than the one satisfied by the single vector function $\phi^{(k)}$. For some examples on the use of higher-order variational equations see e.g. [75].

Although the variational equation $V E_{k}$ is not a linear differential equation, it is in fact equivalent to one: there exists a linear differential equation $L V E_{k}$ with coefficients in the field of meromorphic functions over $\Gamma$ (resp. $\bar{\Gamma}$ ) such that the differential extensions generated by the solutions of $V E_{k}$ coincide with the Picard-Vessiot extensions of $L V E_{k}$. This leads to the consideration of the Galois group $G_{k}$ of $V E_{k}$, i.e., of the $L V E_{k}$, naturally justifying our attempt at generalising Theorem 1 to the higher-order variational equations. Indeed, it was already conjectured in [59, Chapter 8], by the first author, that a necessary condition for complete integrability of the Hamiltonian system (1) by means of meromorphic first integrals is that the identity component $\left(G_{k}\right)^{0}$ be commutative for any $k \geqslant 1$. The present paper is devoted to proving this conjecture. This result was announced in [60] and our proof essentially follows along the same lines as the one involving the first-order variational equation in [62]. A completely new tool, however, will be central here, namely a flatness argument in the context of completion of analytical power series. Not only does this argument replace Ziglin's lemma; it actually yields a new proof of the first-order case (Theorem 1) without the use of said lemma.

We will now state our main results. The precise definitions and complete proofs will follow later. As before we consider a non stationary particular solution $\Gamma$ of (1); for simplicity we "identify" the abstract Riemann surface $\Gamma$ with its immersion $\iota \Gamma$ in $M$. For each point $m \in$ $\Gamma \subset M$, there is a natural faithful representation of the Galois group $G_{k}$ of the $k$-th variational equation $V E_{k}$ in the group of $k$-jets at $m$ of symplectic diffeomorphisms $\operatorname{Diff}_{S p}^{k}(M, m)$ fixing $m$. We will identify the groups $G_{k}$ with their images.

$4^{\text {e }}$ SÉRIE - TOME $40-2007-\mathrm{N}^{\circ} 6$ 
There are natural group homomorphisms $G_{k+1} \rightarrow G_{k}$ which happen to be surjective as a consequence of the differential Galois correspondence. We introduce the inverse limit $\hat{G}=$ $\lim _{k} G_{k}$, which is a pro-algebraic group endowed with the Zariski topology. We can identify it

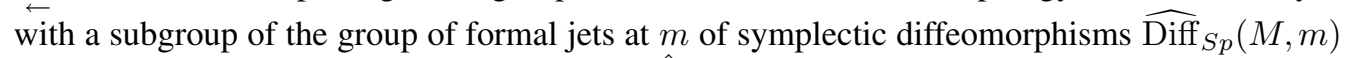
fixing $m$. We have then surjective morphisms $\hat{G} \rightarrow G_{k}$, for $k \in \mathbf{N}^{*}$.

Proposition 1. - Given $k \in \mathbf{N}^{*}$ :

(i) we have natural isomorphisms of finite groups

$$
\begin{gathered}
G_{k} /\left(G_{k}\right)^{0} \rightarrow G_{1} /\left(G_{1}\right)^{0}, \\
\hat{G} / \hat{G}^{0} \rightarrow G_{1} /\left(G_{1}\right)^{0} ;
\end{gathered}
$$

(ii) $G_{k}$ (resp. $\left.\hat{G}\right)$ is Zariski connected if and only if $G_{1}$ is Zariski connected;

(iii) $\left(G_{k}\right)^{0}$ (resp. $\left.\hat{G}^{0}\right)$ is solvable if and only if $\left(G_{1}\right)^{0}$ is solvable. In particular if $\left(G_{1}\right)^{0}$ is commutative, then $\left(G_{k}\right)^{0}$ and $\hat{G}^{0}$ are solvable.

THEOREM 2. - If the Hamiltonian system (1) is completely integrable with meromorphic first integrals in a neighbourhood of $\Gamma$, not necessarily independent on $\Gamma$ itself, then:

(i) for each $k \in \mathbf{N}^{*}$ the identity component $\left(G_{k}\right)^{0}$ of the Galois group $G_{k}$ of the $k$-th variational equation $V E_{k}$ is commutative;

(ii) for each $k \in \mathbf{N}^{*}$ the Galois group of the equation $V E_{k}$ is virtually commutative;

(iii) for each $k \in \mathbf{N}^{*}$ the Lie algebra $\mathcal{G}_{k}$ of the Galois group $G_{k}$ of the $k$-th variational equation $V E_{k}$ is abelian;

(iv) the identity component $\hat{G}^{0}$ of the group $\hat{G}$ is commutative;

(v) the group $\hat{G}^{0}$ is virtually commutative;

(vi) the Lie algebra $\hat{\mathcal{G}}$ of the group $\hat{G}^{0}$ is abelian.

We remark that if there is no obstruction to integrability at the first level, meaning $\left(G_{1}\right)^{0}$ is commutative and thus Theorem 1 fails to prove non-integrability, we can then try to find a $k>1$ such that the group $G_{k}^{0}$ is non-commutative. This group will automatically be solvable, however. Therefore, notably, Hamiltonian non-integrability will be countered by Picard-Vessiot integrability of the variational equations.

As was the case for Theorem 1, we obtain valid variants of our current main result when adding some distinguished points to $\Gamma$. In such situations we have the following result.

Proposition 2. - Let $k \in \mathbf{N}^{*}$. Then the $k$-th variational equation is regular singular if and only if the first variational equation is regular singular.

If we do not add points to $\Gamma$ then all the $V E_{k}$ are regular singular because they correspond to holomorphic connections.

From the preceding facts, we can derive purely topological results on the dynamics of integrable Hamiltonian systems, extending Ziglin's results (cf. also [36]).

Let $\gamma$ be a continuous closed loop of $\Gamma$ at $m \in \Gamma$. The flow of the Hamiltonian system (1) near $\gamma$ will give a germ $\psi_{\gamma} \in \operatorname{Diff}_{S p}(M, m)$ of the group of germs of analytic symplectic diffeomorphisms. We call it the holonomy of $\gamma$. Using the time parametrization, we can interpret the time "along $\gamma$ " as a time translation. If we continuously deform the closed loop $\gamma$ at $m$, then the germ $\psi_{\gamma}$ will not change, the immediate outcome being a group homomorphism

$$
\rho: \pi_{1}(\Gamma, m) \rightarrow \operatorname{Diff}_{S p}(M, m)
$$

called the holonomy representation. Note that we must take the opposite group law on the fundamental group $\pi_{1}(\Gamma, m)$. 
We have natural maps

$$
\operatorname{Diff}_{S p}(M, m) \rightarrow \widehat{\operatorname{Diff}}_{S p}(M, m)
$$

Identifying Diff ${ }_{S p}(M, m)$ with a subgroup of $\widehat{\operatorname{Diff}}_{S p}(M, m)$, we obtain $\operatorname{Im} \rho \subset \hat{G}$ and the $k$-jets of the holonomies, $\rho_{k}$, satisfy $\operatorname{Im} \rho_{k} \subset G_{k}$. Adding equilibrium points and points at infinity to $\Gamma$ does not change the holonomy groups but can change the Galois groups, however.

COROLlARY 2. - If the Hamiltonian system (1) is completely integrable with meromorphic first integrals in a neighbourhood of $\Gamma$, not necessarily independent on $\Gamma$ itself, then, denoting by $\operatorname{Im} \rho$ the holonomy group associated to a solution $\Gamma$ :

(i) $\operatorname{Im} \rho \subset \hat{G}$ is virtually commutative;

(ii) for $k \in \mathbf{N}^{*}$, the groups of $k$-jets $\operatorname{Im} \rho_{k} \subset G_{k}$ are virtually commutative;

(iii) $\operatorname{Im} \rho$ is Zariski dense in $\hat{G}$, and for $k \in \mathbf{N}^{*}$, the group $\operatorname{Im} \gamma_{k}$ is Zariski dense in $G_{k}$. Statement (iii) remains true when adding some points to $\Gamma$ if we assume, moreover, that the meromorphic extension of the first variational equation to the completed curve is regular singular.

As for the case of the first variational equations, we can, in the case of higher variational equations, "eliminate the trivial solutions". We can moreover restrict ourselves to the energy hypersurface $M_{0}$ containing our solution $\Gamma$. This is where we obtain the normal variational equations of higher order. Choosing a small fibration transversal to the flow $M_{1}$ in $M_{0}$, the corresponding Galois groups are subgroups of $k$-jets on $M_{1}$ at $m$. For these groups we have an evident version of our main theorem, that is Theorem 2.

There is also a topological version: in Corollary 2, we can replace the holonomy group of the flow by the holonomy group of the corresponding one-dimensional foliation, or, in order to keep the symplectic property, the holonomy of the one-dimensional foliation of the Hamiltonian system restricted to the energy hypersurface.

\section{Jets and variational equations}

\subsection{Jets and jet groups}

We will make an essential use of the jet formalism due to C. Ehresmann. We recall the basic definitions and results here. Our references are [19, Chapter 1, paragraph 3], [40,39,58] or the paper [77] where the reader can find more details. In general the jet formalism is described for $\mathcal{C}^{\infty}$ real functions. Here we will only use complex holomorphic functions.

As usual, given a manifold $M$ and a point $p \in M$, we denote as $(M, p)$ the germ of the manifold $M$ at $p$. Let $f, g:(\mathbf{C}, 0) \rightarrow \mathbf{C}$ be two germs of holomorphic functions at the origin and $k \in \mathbf{N}$. We assume that $f(0)=g(0)$. We will say that $f$ and $g$ have the same $k$-jet at 0 if $f^{(j)}(0)=g^{(j)}(0)$ for $j \leqslant k$.

Now let $M, N$ be complex analytic manifolds, and let $p \in M, q \in N$. Let $f: M \rightarrow N$ and $g: M \rightarrow N$ be holomorphic maps of $M$ into $N$. We will say that $f$ and $g$ have the same $k$-jet at $p$ whenever

- $f(p)=g(p)=q$,

- for all $p$-based parametrized analytic curves: $v:(\mathbf{C}, 0) \rightarrow(M, p)$ and all $q$-based complex valued holomorphic functions $u: N \rightarrow \mathbf{C}$, the holomorphic maps $u \circ f \circ v$ and $u \circ g \circ v$ have the same $k$-jet. It is an equivalence relation and the corresponding equivalence class will be denoted by $j_{p}^{k}(f)$. The point $p$ is the source of $j_{p}^{k}(f)$ and the point $q$ is its target.

We need to clarify the implications of the preceding definition in local coordinates.

$4^{\text {e }}$ SÉRIE - TOME $40-2007-\mathrm{N}^{\circ} 6$ 
Let $U$ be an open neighbourhood of 0 in $\mathbf{C}^{m}$. Let $f, g$ be two differentiable maps $f, g: U \rightarrow$ $\mathbf{C}^{n}$, with $f(0)=g(0)=0$. In coordinates $x=\left(x_{1}, \ldots, x_{m}\right), f(x)=\left(f_{1}(x), \ldots, f_{n}(x)\right), g(x)=$ $\left(g_{1}(x), \ldots, g_{n}(x)\right)$. We will use the classical notations for partial derivatives: for a multi-index $\mu=\left(\mu_{1}, \ldots, \mu_{m}\right), D_{x}^{\mu}=D^{\mu}=\frac{\partial^{\mu_{1}}}{\partial x_{1}^{\mu_{1}}} \cdots \frac{\partial^{\mu_{m}}}{\partial x_{m}^{\mu_{m}}}$. Then $f$ and $g$ have the same $k$-jet at the origin if and only if

$$
D_{x}^{\mu} f_{i}(0)=D_{x}^{\mu} g_{i}(0), \quad 1 \leqslant i \leqslant n,|\mu|=\sum_{i=1}^{m} \mu_{i} \leqslant k .
$$

Let $J_{p, q}^{k}(M, N)$ denote the set of all $k$-jets of maps from $M$ to $N$ of source $p$ and target $q$. We define the set

$$
J^{k}(M, N)=\bigcup_{p \in M, q \in N} J_{p, q}^{k}(M, N) .
$$

We have the classical source and target projections

$$
\alpha: J^{k}(M, N) \rightarrow M, \quad \beta: J^{k}(M, N) \rightarrow N,
$$

defined by $\alpha\left(j_{p}^{k}(f)\right)=p$ and $\beta\left(j_{p}^{k}(f)\right)=f(p)=q$.

Now let $\left\{U_{i}\right\}_{i \in I}$ and $\left\{V_{j}\right\}_{j \in J}$ be, respectively, open coordinates coverings of $M$ and $N$. We then have an open covering $\left\{W_{i j}\right\}_{i \in I, j \in J}$ of $J^{k}(M, N)$ :

$$
W_{i j}=\left\{j_{p}^{k}(f) \mid \alpha\left(j_{p}^{k}(f)\right) \in U_{i}, \beta\left(j_{p}^{k}(f)\right) \in V_{j}\right\} .
$$

If $\left\{x_{1}, \ldots, x_{m}\right\}$ and $\left\{y_{1}, \ldots, y_{n}\right\}$ are the coordinate functions on $U_{i}$ and $V_{j}$, respectively, we can define coordinate functions (called natural coordinates) on $W_{i j}$ by

$$
\left(x_{i}(p), y_{j}(q), D_{x}^{\mu}\left(y_{j} \circ f\right)(p)\right), \quad 1 \leqslant i \leqslant m, 1 \leqslant j \leqslant n, 1 \leqslant|\mu| \leqslant k .
$$

Holomorphic changes of local coordinates in $U_{i}$ and $V_{j}$ induce holomorphic changes of coordinates in $W_{i j}$. Hence, we have a complex analytic structure on $J^{k}(M, N)$. If $\operatorname{dim} M=m$ and $\operatorname{dim} N=n$, then

$$
\operatorname{dim} J^{k}(M, N)=m+n\left(\begin{array}{c}
m+k \\
k
\end{array}\right) .
$$

We have $\operatorname{dim} J^{k}(M, \mathbf{C}):=\nu_{m, k}=m+\left(\begin{array}{c}m+k \\ k\end{array}\right)$. If $m$ is already fixed, we set $\nu_{m, k}=\nu_{k}$.

Examples. - The cotangent bundle $T^{*}(M)$ is identified with $J^{1}(M, \mathbf{C})$ and the tangent bundle $T(M)$ is identified with $J^{1}(\mathbf{C}, M)$.

For $r \leqslant k$ there is a natural map $\pi_{k, r}: J_{p, q}^{k}(M, N) \rightarrow J_{p, q}^{r}(M, N)$.

Let $M_{1}, M_{2}, M_{3}$ be three complex analytic manifolds. Let $p_{i} \in M_{i}, i=1,2,3$. The composition of applications induces an algebraic map

$$
J_{p_{2}, p_{3}}^{k}\left(M_{2}, M_{3}\right) \times J_{p_{1}, p_{2}}^{k}\left(M_{1}, M_{2}\right) \rightarrow J_{p_{1}, p_{3}}^{k}\left(M_{1}, M_{3}\right) .
$$

This follows from the chain rule, which allows for the expression of the partial derivatives of a composition map $g \circ f$ as polynomials in the partial derivatives of $g$ and $f$.

In the special case $M_{1}=M_{2}=M_{3}=M, p_{1}=p_{2}=p_{3}=p$, this composition map induces a product in $J_{p, p}^{k}(M, M)$. We will denote by $\operatorname{Diff}^{k}(M, p)$ the subset of $J_{p, p}^{k}(M, M)$ of 
invertible elements. There is a natural isomorphism $\operatorname{Diff}^{1}(M, p) \simeq \mathrm{GL}\left(T_{p}(M)\right)$. In particular, $\operatorname{Diff}^{1}\left(\mathbf{C}^{m}, 0\right) \simeq \mathrm{GL}(m ; \mathbf{C})$.

We set $J_{p, 0}^{k}(M, \mathbf{C})=J^{k}(M, p)$. The group $\operatorname{Diff}^{k}(M, p)$ acts linearly on $J^{k}(M, p)$ by composition on the right. The corresponding representation is faithful. In particular we have a linear action of $\operatorname{Diff}{ }^{k}\left(\mathbf{C}^{m}, 0\right)$ on $J^{k}\left(\mathbf{C}^{m}, 0\right)$ and a faithful representation of $\operatorname{Diff}^{k}\left(\mathbf{C}^{m}, 0\right)$ (or more precisely of the opposite group) into $\operatorname{GL}\left(J^{k}\left(\mathbf{C}^{m}, 0\right)\right)$.

Using local coordinates we obtain the obvious identifications $J^{k}(M, p) \simeq J^{k}\left(\mathbf{C}^{m}, 0\right)$, $J_{p, p}^{k}(M, M) \simeq J_{0,0}^{k}\left(\mathbf{C}^{m}, \mathbf{C}^{m}\right), \operatorname{Diff}^{k}(M, p) \simeq \operatorname{Diff}^{k}\left(\mathbf{C}^{m}, 0\right)$.

The $\mathbf{C}$-algebra structure of $\mathbf{C}$ gives a $\mathbf{C}$-algebra structure on $J^{k}\left(\mathbf{C}^{m}, 0\right)$ and the maps $\pi_{k, r}$ are surjective homomorphisms of $\mathbf{C}$-algebras. The linear action of $\operatorname{Diff}^{k}\left(\mathbf{C}^{m}, 0\right)$ on $J^{k}\left(\mathbf{C}^{m}, 0\right)$ gives an automorphism of $\mathbf{C}$-algebras of $J^{k}\left(\mathbf{C}^{m}, 0\right)$. More precisely, we have the following result.

PROPOSITION 3. - Let $\Phi$ be a linear endomorphism of $J^{k}\left(\mathbf{C}^{m}, 0\right)$. The following conditions are equivalent:

(i) $\Phi$ is an homomorphism of $\mathbf{C}$-algebras.

(ii) There exists $\phi \in J_{0,0}^{k}\left(\mathbf{C}^{m}, \mathbf{C}^{m}\right)$ such that $\Phi(Y)=Y \circ \phi$ for all $Y \in J^{k}\left(\mathbf{C}^{m}, 0\right)$.

Moreover, if these conditions are satisfied, then $\Phi$ is an automorphism of $\mathbf{C}$-algebras if and only if $\phi \in \operatorname{Diff}^{k}\left(\mathbf{C}^{m}, 0\right)$.

Proof. - The implication (ii) $\Rightarrow$ (i) is trivial. It remains to prove (i) $\Rightarrow$ (ii). Let $x_{1}, \ldots, x_{m}$ be the coordinate functions in $\mathbf{C}^{m}$. We denote the corresponding jets in $J^{k}\left(\mathbf{C}^{m}, 0\right)$ in identical manner. Then we set $\phi_{i}=\Phi\left(x_{i}\right)$.

Let $Y \in J^{k}\left(\mathbf{C}^{m}, 0\right)$. We can write $Y$ as an element of $\mathbf{C}\left[x_{1}, \ldots, x_{m}\right]: Y=P\left(x_{1}, \ldots, x_{m}\right)$. Then $\Phi(Y)=\Phi\left(P\left(x_{1}, \ldots, x_{m}\right)\right)=P\left(\Phi\left(x_{1}\right), \ldots, \Phi\left(x_{m}\right)\right)=P\left(\phi_{1}, \ldots, \phi_{m}\right)=P \circ \phi=$ $Y \circ \phi$.

The group $\operatorname{Diff}^{k}\left(\mathbf{C}^{m}, 0\right)$ is a linear complex algebraic group. We have an exact sequence of algebraic groups:

$$
\{\mathrm{id}\} \rightarrow I^{k}\left(\mathbf{C}^{m}, 0\right) \rightarrow \operatorname{Diff}^{k}\left(\mathbf{C}^{m}, 0\right) \stackrel{\pi_{k, 1}}{\longrightarrow} \mathrm{GL}(m ; \mathbf{C}) \rightarrow\{\mathrm{id}\}
$$

where $I^{k}\left(\mathbf{C}^{m}, 0\right)$ is the subgroup of $\operatorname{Diff}^{k}\left(\mathbf{C}^{m}, 0\right)$ of germs tangent to identity. Using coordinates it is easy to build a section of the homomorphism $\pi_{k, 1}$. Therefore $\operatorname{Diff}^{k}\left(\mathbf{C}^{m}, 0\right)$ is a semi-direct product of $\mathrm{GL}(m ; \mathbf{C})$ by the unipotent group $I^{k}\left(\mathbf{C}^{m}, 0\right)$. More precisely we have exact sequences of algebraic groups

$$
\{\mathrm{id}\} \rightarrow I^{k+1, k}\left(\mathbf{C}^{m}, 0\right) \rightarrow I^{k+1}\left(\mathbf{C}^{m}, 0\right) \rightarrow I^{k}\left(\mathbf{C}^{m}, 0\right) \rightarrow\{\mathrm{id}\}
$$

$I^{k+1, k}\left(\mathbf{C}^{m}, 0\right)$ being the vector group in $I^{k+1}\left(\mathbf{C}^{m}, 0\right)$ of elements with only non-trivial contributions of order $k+1$, i.e., $I^{k+1}\left(\mathbf{C}^{m}, 0\right)$ is the semi-direct product of $I^{k}\left(\mathbf{C}^{m}, 0\right)$ by the additive group of a finite-dimensional vector space. Therefore we obtain $\operatorname{Diff}^{k}\left(\mathbf{C}^{m}, 0\right)$ from $\mathrm{GL}(m ; \mathbf{C})$ by a sequence of semi-direct products with additive groups of finite-dimensional vector spaces $[39,77]$.

We need some symplectic variations. We denote $(q, p)=\left(q_{1}, \ldots, q_{n}, p_{1}, \ldots, p_{n}\right) \in \mathbf{C}^{2 n}$, and we set $\Omega=d q \wedge d p=\sum_{i=1}^{n} d q_{i} \wedge d p_{i}$. We will denote by $\operatorname{Diff}_{S p}^{k}\left(\mathbf{C}^{2 n}, 0\right)$ the subgroup of jets of diffeomorphisms $\phi$ such that $\phi * \Omega=\Omega$, in the evident sense. This subgroup is clearly an algebraic subgroup. It is easy to check that, for $k \geqslant r$, the homomorphisms of group $\pi_{k, r}$ induce a surjective homomorphism of groups

$$
\pi_{k, r}: \operatorname{Diff}_{S p}^{k}\left(\mathbf{C}^{2 n}, 0\right) \rightarrow \operatorname{Diff}_{S p}^{r}\left(\mathbf{C}^{2 n}, 0\right) .
$$

$4^{\text {e }}$ SÉRIE - TOME $40-2007-\mathrm{N}^{\circ} 6$ 
We easily see that $\operatorname{Diff}_{S p}^{k}\left(\mathbf{C}^{2 n}, 0\right)$ is the semi-direct product of $S p(2 n ; \mathbf{C})$ by a unipotent group. More precisely we obtain $\operatorname{Diff}_{S p}^{k}\left(\mathbf{C}^{2 n}, 0\right)$ from $S p(2 n ; \mathbf{C})$ by a sequence of semi-direct products with additive groups of finite-dimensional vector spaces.

It is easy to extend the preceding definitions when we replace $\left(\mathbf{C}^{2 n}, 0\right)$ by a complex symplectic manifold germ $(M, m)=((M, \Omega), m)$. We shall denote by $\operatorname{Diff}_{S p}^{k}(M, m)$ the subgroup of germs of symplectic diffeomorphisms in $\operatorname{Diff}(M, m)$, etc.

\subsection{Relations and flatness}

In the statement of the central result of the next Section 3.3, namely Theorem 3, we will use formal power series of several complex variables. This is essential for the application to higher variational equations, the convergent case being insufficient. In the proof of Theorem 3, it is necessary to extend a result which is true for convergent power series "for geometric reasons" to a similar result for formal power series. The flatness of the ring of formal power series on the ring of convergent power series is a classical tool in order to handle such problems.

The important result for the application in the next section is Corollary 3. There exists a more general and difficult version of this result, Artin's theorem [7], allowing nonlinear equations. Readers acquainted with this latter result may skip the remainder of this section and apply it directly in the next one.

We will use some definitions and results from [8, Chapters 2 and 10] (cf. also [18, Chapter 1] or [73, Annexe, p. 34]). Henceforth, all rings will be assumed to be commutative and unitary and all modules will be assumed unitary.

Definition 1. - Let $A$ be a ring and let $M$ be a $A$-module. We say $M$ is $A$-flat (or flat) if, for every $A$-module $N$ and every submodule $N^{\prime}$ of $N$, the natural map

$$
M \otimes_{A} N^{\prime} \rightarrow M \otimes_{A} N
$$

is injective.

This definition is equivalent to the following property.

(R) Let $a_{1}, \ldots, a_{p} \in A$ and $\left(m_{1}, \ldots, m_{p}\right) \in M^{p}$ a relation between $a_{i}, i=1, \ldots, p$ with coefficients in $M$ (i.e. $\left.\sum_{i=1}^{p} a_{i} m_{i}=0\right)$. Then there exist $b_{i j} \in A(i=1, \ldots, p, j=$ $1, \ldots, q ; q \in \mathbf{N})$ and $n_{j} \in M$ such that:

(i) for every $j,\left(b_{1 j}, \ldots, b_{p j}\right)$ is a relation between $a_{i}, i=1, \ldots, p$;

(ii) for every $i, m_{i}=\sum_{j=1}^{q} b_{i j} n_{j}$.

Property (R) essentially means that relations between $a_{1}, \ldots, a_{p}$ with their coefficients in $M$ are generated by relations having coefficients in $A$.

We recall that if $M$ is $A$-flat, for every exact sequence of $A$-modules $N^{\prime} \rightarrow N \rightarrow N^{\prime \prime}$, the sequence $M \otimes_{A} N^{\prime} \rightarrow M \otimes_{A} N \rightarrow M \otimes_{A} N^{\prime \prime}$ is exact.

PROPOSITION 4. - The ring of formal power series $\hat{A}=\mathbf{C}\left[\left[x_{1}, \ldots, x_{n}\right]\right]$ is a flat module on its subring of convergent power series $A=\mathbf{C}\left\{x_{1}, \ldots, x_{n}\right\}$.

Proof. - We denote by $\mathcal{M}$ the maximal ideal of $A$, i.e., the elements of $A$ without zeroorder term; $\hat{A}$ is the completed ring of the local ring $A$ relatively to the $\mathcal{M}$-adic topology. $A$ is Noetherian in virtue of Hilbert's basis theorem. Then Proposition 10.14 of [8] implies $\hat{A}$ is flat.

In an entirely analogous manner, we denote by $\hat{\mathcal{M}}$ the maximal ideal of $\hat{A}$. 
Corollary 3. - We set, as above, $A=\mathbf{C}\left\{x_{1}, \ldots, x_{n}\right\}$ and $\hat{A}=\mathbf{C}\left[\left[x_{1}, \ldots, x_{n}\right]\right]$. We consider an analytic system of $m$ linear equations, with $p$ unknowns $t_{1}, \ldots, t_{p}$ :

$$
a_{1 h} t_{1}+\cdots+a_{p h} t_{p}=0 \quad\left(h=1, \ldots, m, a_{i h} \in A\right) .
$$

We assume this system admits a formal solution $\hat{T}=\left(\hat{t}_{1}, \ldots, \hat{t}_{p}\right) \in \hat{A}^{p}$. Then, for every $\mu \in \mathbf{N}$, the system admits an analytic solution $\left(t_{1}^{(\mu)}, \ldots, t_{p}^{(\mu)}\right) \in A^{p}$ such that, for $i=1, \ldots, p, \hat{t}_{i}=t_{i}^{(\mu)}$ (mod. $\left.\hat{\mathcal{M}}^{\mu+1}\right)$.

Proof. - We denote $\Phi: A^{p} \rightarrow A^{m}$ (resp. $\hat{\Phi}: \hat{A}^{p} \rightarrow \hat{A}^{m}$ ) the $A$-linear (resp. $\hat{A}$-linear) map defined by the matrix $a=\left(a_{i h}\right)$.

Using Proposition 4, we have $\operatorname{Ker} \hat{\Phi}=\operatorname{Ker} \Phi \otimes_{A} \hat{A}$. Therefore the formal solutions of the system (5) are generated, over $\hat{A}$, by the analytical solutions. Then, if $\hat{T}=\left(\hat{t}_{1}, \ldots, \hat{t}_{p}\right)$ is a formal solution, there exist $\hat{n}_{1}, \ldots \hat{n}_{q} \in \hat{A}$ and $q$ analytic solutions $T_{j}=\left(t_{1 j}, \ldots, t_{p j}\right)(j=1, \ldots, q)$, such that $\left(\hat{t}_{1}, \ldots, \hat{t}_{p}\right)=\sum_{j=1}^{q} \hat{n}_{j} T_{j}$. Replacing the $\hat{n}_{j}$ by their $k$-jets $n_{j}^{(k)}$ (which we interpret as polynomials in the variables $\left.x_{1}, \ldots, x_{n}\right)$, we obtain an analytic solution $T^{(k)}=\sum_{j=1}^{q} n_{j}^{(k)} T_{j}$ of (5). If we choose $k=\mu$, then $T^{(\mu)}=\left(t_{1}^{(\mu)}, \ldots, t_{p}^{(\mu)}\right)$ and $\hat{T}$ have the same $\mu$-jet .

\subsection{Some properties of Poisson algebras}

In this section we describe some properties of the Poisson algebras of germs of meromorphic and formal meromorphic functions defined on a neighbourhood of the origin of a complex symplectic vector space.

Let $E$ be a complex symplectic vector space of complex dimension $2 n$. The symplectic product is denoted by $\omega:(v, w) \mapsto \omega(v, w)$. Using the unique 2 -form $\Omega$ invariant by translation and satisfying $\Omega(0)=\omega$ over $E$, we will consider $E$ as a symplectic manifold. We can choose symplectic coordinates $(q, p)$ over $E$ and thus achieve $\Omega=\sum_{i=1, \ldots, n} d p_{i} \wedge d q_{i}$.

We will consider the $\mathbf{C}$-algebras of polynomials, convergent series and formal series over $E$ : $\mathbf{C}[E], \mathbf{C}\{E\}, \mathbf{C}[[E]]$, and the corresponding fraction fields $\mathbf{C}(E), \mathbf{C}(\{E\}), \mathbf{C}((E))$, respectively. On all these algebras we have a Poisson product

$$
\{f, g\}=\Omega\left(d f^{\sharp}, d g^{\sharp}\right) .
$$

We consider the spaces of germs at the origin of vector fields $X$ of different kinds: holomorphic, meromorphic, formal, formal-meromorphic. That is $X=\sum_{i} a_{i} \frac{\partial}{\partial q_{i}}+\sum_{i} b_{i} \frac{\partial}{\partial p_{i}}, a_{i}, b_{i} \in$ $\mathbf{C}\{E\}, \mathbf{C}(\{E\}), \mathbf{C}[[E]], \mathbf{C}((E))$, respectively.

The corresponding complex vector spaces are endowed with the Lie algebra structures defined by the usual bracket of vector fields. A germ of a vector field $X$ of one of the previous kinds is said to be Hamiltonian if there exists a germ of a function $f$ of the same kind such that

$$
X=d f^{\sharp} .
$$

Then we will denote $X=X_{f}$.

The following results are well known [1] (3.36, Proposition, page 189).

Proposition 5. - For a germ of a vector field $X$ of one of the above kinds, the following conditions are equivalent:

(i) $X$ is Hamiltonian;

(ii) $d\left(X^{b}\right)=0$;

$4^{\text {e }}$ SÉRIE - TOME $40-2007-\mathrm{N}^{\circ} 6$ 
(iii) $L_{X} \Omega=0$;

(iv) $(\exp X)^{*} \Omega=\Omega$.

PROPOSITION 6. - The germs of Hamiltonian vector fields of any of the kinds above are a Lie subalgebra by defining

$$
\left[X_{f}, X_{g}\right]=X_{\{f, g\}} .
$$

Let $((M, \Omega), m)$ be a germ of a complex analytic symplectic manifold $\left(\operatorname{dim}_{\mathbf{C}} M=2 n\right)$. We recall that the natural maps

$$
\operatorname{Diff}^{k+1}(M, m) \rightarrow \operatorname{Diff}^{k}(M, m), \quad \operatorname{Diff}_{S p}^{k+1}(M, m) \rightarrow \operatorname{Diff}_{S p}^{k}(M, m)
$$

are surjective morphisms of algebraic groups.

Taking inverse limits we obtain the pro-algebraic group $\widehat{\operatorname{Diff}}(M, m)=\lim _{k} \operatorname{Diff}^{k}(M, m)$ and the pro-algebraic subgroup $\widehat{\operatorname{Diff}}_{S p}(M, m)=\lim _{k} \operatorname{Diff}_{S p}^{k}(M, m)$. These pro-algebraic groups are endowed with the Zariski topology, which by definition happens to be the direct limit topology. We have surjective morphisms

$$
\widehat{\operatorname{Diff}}(M, m) \rightarrow \operatorname{Diff}^{k}(M, m), \quad \widehat{\operatorname{Diff}}_{S p}(M, m) \rightarrow \operatorname{Diff}_{S p}^{k}(M, m)
$$

A $k$-jet at $m \in M$ of a vector field $X$ vanishing at $m$ acts linearly on the space $J^{k}(M, m)$ of $k$-jets of functions by truncation of the Lie derivative $L_{X}$. Then $\exp X=\exp L_{X}$ can be interpreted as an element of $\operatorname{Diff}^{k}(M, m)$. It is easy to check that, using this remark, we can identify the space $\mathcal{L}^{k}(M, m)$ of $k$-jets of vector fields vanishing at $m$ with the Lie algebra of the algebraic group $\operatorname{Diff}^{k}(M, m)$. Then $\widehat{\mathcal{L}}(M, m)=\lim _{k} \mathcal{L}^{k}(M, m)$ is identified with the Lie algebra of the pro-algebraic group $\widehat{\operatorname{Diff}}(M, m)$. Beware of the fact that the diffeomorphisms $\exp \hat{X}$ can be analytic, that is $\exp \hat{X} \in \operatorname{Diff}(M, m)$, even when $\hat{X}$ is divergent.

Similarly we can identify the space $\mathcal{L}_{s p}^{k}(M, m)$ of $k$-jets of Hamiltonian vector fields vanishing at $m$ with the Lie algebra of the algebraic group $\operatorname{Diff}_{S p}^{k}(M, m)$. Then $\widehat{\mathcal{L}}_{s p}(M, m)=$ $\lim _{k} \mathcal{L}_{s p}^{k}(M, m)$ is identified with the Lie algebra of the pro-algebraic group $\widehat{\operatorname{Diff}}_{S p}(M, m)$. There is a $k$-truncated version of Proposition 5. We leave the details to the reader.

It is possible to define Poisson products on spaces of germs of $k$-jets $J^{k}(M, m)$ :

$$
\begin{gathered}
J^{k}(M, m) \times J^{k}(M, m) \rightarrow J^{k-1}(M, m), \quad k>0, \\
J^{k, 2}(M, m) \times J^{k, 2}(M, m) \rightarrow J^{k, 2}(M, m), \quad k>1,
\end{gathered}
$$

where $J^{k, 2}(M, m)$ is the space of jets vanishing at order one. We say an endomorphism of $J^{k}(M, m)$ preserves the Poisson product if it preserves these Poisson products.

There is a symplectic version of Proposition 3:

Proposition 7. - Let $\Phi$ be a linear endomorphism of $J^{k}(E, 0)$. The following conditions are equivalent:

(i) $\Phi$ is an homomorphism of $\mathbf{C}$-algebras and preserves the Poisson product;

(ii) there exists $\phi \in J_{S p}^{k}(E, E)$ such that $\Phi(Y)=Y \circ \phi$ for all $Y \in J^{k}(E, 0)$.

Moreover if these conditions are satisfied, then $\Phi$ is an automorphism of $\mathbf{C}$-algebras if and only if $\phi \in \operatorname{Diff}_{S p}^{k}(E, 0)$.

We will now state and prove the main result of this section, which is also the central tool in our obstruction theorem. It is also crucial to the analogue result in non-linear Galois theory, due to the 
second author (cf. Section 5 below). Since the result is local, we can choose Darboux coordinates in a neighbourhood of $m$ in $M$, and it is sufficient to study the case $(M, m)=(E, 0)$. We will denote $\operatorname{Diff}^{k}(E, 0), \ldots$ the corresponding objects.

Let $f_{1}, \ldots, f_{\ell}$ be $\ell$ germs of meromorphic functions (resp. formal meromorphic functions) at $m \in M$. We say they are functionally independent near $m$ if the germ at $m$ of $d f_{1} \wedge \cdots \wedge d f_{\ell}$ is not identically zero. In our hypotheses for the meromorphic case, we pose no obstacle to either the existence of a singularity at $m$ for some $f_{i}$ or the vanishing of $d f_{1} \wedge \cdots \wedge d f_{\ell}$ at $m$, even if $f_{i}$ are holomorphic at $m$.

The following result is easy to prove.

LEMMA 1. - Let $f_{1}, \ldots, f_{\ell}$ be $\ell$ germs of meromorphic functions (resp. formal meromorphic functions) at $m \in M$ which are functionally independent near $m$. Let $\alpha$ be a germ of a meromorphic one-form (resp. a formal meromorphic one-form) at $m$ such that $\alpha \wedge d f_{1} \wedge \cdots \wedge$ $d f_{\ell} \equiv 0$. Then there exist germs of meromorphic functions (resp. formal meromorphic functions) $\theta_{1}, \ldots, \theta_{\ell}$ such that $\alpha=\sum_{i=1, \ldots, \ell} \theta_{i} d f_{i}$.

THEOREM 3. - Let $E$ be a complex symplectic vector space of complex dimension $2 n$. Let $f_{1}, \ldots, f_{n}$ be $n$ germs at the origin of $E$ of meromorphic functions, functionally independent near the origin though not necessarily at the origin itself. Assume these germs $f_{1}, \ldots, f_{n}$ are in pairwise involution and let $\hat{\mathcal{L}}$ be a Lie algebra of Hamiltonian formal vector fields at the origin of $E$. Assume $f_{1}, \ldots, f_{n}$ are invariant by $\hat{\mathcal{L}}$. Then the Lie algebra $\hat{\mathcal{L}}$ is abelian.

Theorem 3, a particular case of which was suggested some years ago to the second author by L. Gavrilov in relation to Theorem 1, is a corollary of the following.

THEOREM 4. - Let $E$ be a complex symplectic vector space of complex dimension $2 n$. Let $f_{1}, \ldots, f_{n}$ be $n$ germs at the origin of $E$ of meromorphic functions, functionally independent near the origin (not necessarily at the origin itself). Assume these germs $f_{1}, \ldots, f_{n}$ are in involution. Let $\mathcal{A}$ be the $\mathbf{C}$-subalgebra of the field $\mathbf{C}((E))$ generated by $f_{1}, \ldots, f_{n}$. Then

(i) $\mathcal{A}$ is involutive,

(ii) the orthogonal $\mathcal{A}^{\perp}$ of $\mathcal{A}$ in $\mathbf{C}((E))$ is an involutive $\mathbf{C}$-subalgebra of $\mathbf{C}((E))$.

Proof. - We start with a preliminary result.

LEMma 2. - Under the hypotheses of Theorem 4, let $\hat{\varphi} \in \mathcal{A}^{\perp} \subset \mathbf{C}((E))$. Then $\hat{\varphi}, f_{1}, \ldots, f_{n}$ are functionally dependent near the origin, that is

$$
d \hat{\varphi} \wedge d f_{1} \wedge \cdots \wedge d f_{n} \equiv 0 .
$$

We will prove a slightly more general version.

LEMMA 3. - Under the hypotheses of Theorem 4, let $\hat{\alpha}$ be a meromorphic (resp. formal meromorphic) one-form. We set $X=\hat{\alpha}^{b}$. Assume that $d f_{i}(X)=\iota_{X_{f_{i}}} \hat{\alpha}=\hat{\alpha}\left(X_{f_{i}}\right)=\omega\left(X_{f_{i}}, X\right)=0$ for $i=1, \ldots, n$. Then,

$$
\hat{\alpha} \wedge d f_{1} \wedge \cdots \wedge d f_{n} \equiv 0
$$

Proof. - First case. Assume $\hat{\alpha}=\alpha$ is meromorphic. Then, in each open neighbourhood of 0 in $E$, there exists a point $x_{0}$ such that $f_{1}, \ldots, f_{n}$ and $\alpha$ are holomorphic at $x_{0}$ and such that $d f_{1} \wedge \cdots \wedge d f_{n}$ does not vanish at $x_{0}$. These properties remain true in an open neighbourhood $U$ of $x_{0}$. Then, for $x_{1} \in U$, the set $V=V_{x_{1}}=\left\{x \in U \mid f_{1}(x)=f_{1}\left(x_{1}\right), \ldots, f_{n}(x)=f_{n}\left(x_{1}\right)\right\}$ is an analytic submanifold of complex dimension $n$ of $U$. We have $d f_{i}(X)=0, i=1, \ldots, n$. Therefore the analytic vector field $X$ is tangent to $V$. We also have $d f_{i}\left(X_{f_{j}}\right)=0, i, j=1, \ldots, n$,

$4^{\text {e }}$ SÉRIE - TOME $40-2007-\mathrm{N}^{\circ} 6$ 
and the analytic vector fields $X_{f_{i}}, i=1, \ldots, n$, are also tangent to $V$. Since the vectors $X_{f_{1}}(x), \ldots, X_{f_{n}}(x)$ are independent at each point of $x \in U$, they generate the tangent space of $V$ at each point of $V$. We obtain a relation $X=\theta_{1} X_{f_{1}}+\cdots+\theta_{n} X_{f_{n}}$ over $U$, where $\theta_{i}$ are holomorphic. The relation $\alpha=\theta_{1} d f_{1}+\cdots+\theta_{n} d f_{n}$ follows and $\alpha \wedge d f_{1} \wedge \cdots \wedge d f_{n} \equiv 0$ over $U$. Hence the similar relation holds for the germs at the origin by analytic continuation.

Remark. - If we assume that the one-form $\alpha$ is closed, then $\left\{d f_{j}, \alpha\right\}^{\sharp}:=\left[X_{f_{j}}, X\right]=0$. Now we consider the previous relation $X=\theta_{1} X_{f_{1}}+\cdots+\theta_{n} X_{f_{n}}$ over $U$. We have

$$
\left[X_{f_{j}}, X\right]=\sum_{i=1, \ldots, n}\left(L_{X_{f_{j}}} \theta_{i}\right) X_{f_{i}}=0, \quad j=1, \ldots, n
$$

Therefore $L_{X_{f_{j}}} \theta_{i}=0, i, j=1, \ldots, n$ and the analytic functions $\theta_{i}, i=1, \ldots, n$ are constant on each manifold $V_{x_{1}},\left(x_{1} \in U\right)$.

Second case. We assume that $\hat{\alpha}$ is a formal one-form, without singularity at the origin. We set $\hat{\alpha}=\sum_{i} y_{i} d p_{i}+\sum_{i} z_{i} d q_{i}$. Then we can interpret the system of equations

$$
\hat{\alpha}\left(X_{f_{i}}\right)=\sum_{j=1}^{n} z_{j} \frac{\partial f_{i}}{\partial p_{j}}-y_{j} \frac{\partial f_{i}}{\partial q_{j}}=0, \quad i=1, \ldots n,
$$

as a system of equations having $2 n$ unknowns $(y(x), z(x))$ which is linear analytic with respect to $x=\left(q_{1}, \ldots, q_{n}, p_{1}, \ldots, p_{n}\right)$.

Let $\hat{\mathcal{M}} \subset \mathbf{C}[[E]]$ be the maximal ideal, that is, the formal series in $E$ without zero-order term. Then, using Corollary 3, or else Artin's theorem [7] if preferred, for every $\mu \in \mathbf{N}^{*}$ there exists a germ $\beta_{\mu}=\sum_{i} \bar{y}_{i} d p_{i}+\sum_{i} \bar{z}_{i} d q_{i}$ of analytic one-forms such that $(\bar{y}, \bar{z})$ satisfies the same analytic system, i.e. $\beta_{\mu}$ satisfies (8):

$$
\beta_{\mu}\left(X_{f_{i}}\right)=0, \quad i=1, \ldots n
$$

and such that

$$
\hat{\alpha}=\beta_{\mu}\left(\bmod \hat{\mathcal{M}}^{\mu}\right),
$$

i.e. the germs $\alpha$ and $\beta$, their coordinates being $(y, z)$ and $(\bar{y}, \bar{z})$, coincide up to order $\mu-1$ in $x$. Using the result for the first case, we obtain

$$
\beta_{\mu} \wedge d f_{1} \wedge \cdots \wedge d f_{n} \equiv 0
$$

for every $\mu \in \mathbf{N}^{*}$. The formal relation

$$
\hat{\alpha} \wedge d f_{1} \wedge \cdots \wedge d f_{n} \equiv 0
$$

follows easily.

General case. Assume $\hat{\alpha}$ is a formal meromorphic one-form. Then there exist a formal oneform $\hat{\zeta}$ and a non-zero formal power series $\hat{g} \in \mathbf{C}[[E]]$ such that $\hat{g} \hat{\alpha}=\hat{\zeta}$. Hence $\hat{\zeta}$ satisfies a system similar to (8). We can apply the result of the second case to $\hat{\zeta}: \hat{\zeta} \wedge d f_{1} \wedge \cdots \wedge d f_{n}=$ $\hat{g} \hat{\alpha} \wedge d f_{1} \wedge \cdots \wedge d f_{n} \equiv 0$. The result for $\hat{\alpha}$ follows: $\hat{\alpha} \wedge d f_{1} \wedge \cdots \wedge d f_{n} \equiv 0$. 
This ends the proof of the lemmae and we can return to the proof of Theorem 4. Let $\hat{\varphi}, \hat{\psi} \in \mathcal{A}^{\perp}$. From Lemmae 1 and 2 we obtain

$$
d \hat{\varphi}=\sum_{i=1, \ldots, n} \hat{\theta}_{i} d f_{i}
$$

with $\hat{\theta}_{i} \in \mathbf{C}((E))$. Therefore

$$
\{\hat{\varphi}, \hat{\psi}\}=d \hat{\varphi}\left(X_{\hat{\psi}}\right)=\sum_{i=1, \ldots, n} \hat{\theta}_{i} d f_{i}\left(X_{\hat{\psi}}\right)=\sum_{i=1, \ldots, n} \hat{\theta}_{i}\left\{f_{i}, \hat{\psi}\right\}=0 .
$$

This ends the proof of Theorem 4.

It remains to prove Theorem 3. Let $X \in \hat{\mathcal{L}}$. By definition we have $L_{X}\left(f_{i}\right)=0(i=1, \ldots, n)$. There exists a formal power series expansion $\hat{g} \in \mathbf{C}[[E]]$ such that $X^{b}=d \hat{g}$ (the formal field $X$ is Hamiltonian). Then, with the notations of Theorem 3, we have $\hat{g} \in \mathcal{A}^{\perp}$. The result follows easily.

\subsection{Variational equations. Linearised variational equations}

We will briefly recall the definition of the higher-order variational equations $V E_{k}, k>1$. First we shall use local coordinates, giving the equations in a compact form. These equations are clearly non-linear. However, if we start from the solutions of the equations of order $\leqslant k$, then it is possible to solve the equation of order $k+1$ by a quadrature. But it is not a priori evident that the theory of Picard-Vessiot extensions is applicable to the higher-order variational equations. Therefore it is necessary, for our purpose, to introduce an equivalent linearised version of these equations $L V E_{k}$; roughly speaking this is related to the fact that the jet groups are linear groups. The first author described in [59] how to linearise the second and third variational equations ([59], Section 8.3). Here this will be done in a systematic way. First a local version of this linearisation using coordinates is introduced. Later on we will use a geometric interpretation of the corresponding computations (based upon a duality trick) to derive a global version.

The global geometric version is indispensable for the proof. The coordinate version is not needed, strictly speaking, but can be useful for explicit computations in the applicationsfor instance, when using computer algebra or performing numerical checks by means of the variational equations, [49]. See also Appendix B. What follows should ensure users of our main theorem that performing these computations without an explicit need for the linearised variational equations, while at the same time remaining automatically in a convenient PicardVessiot extension (depending on the order $k$ ) of a "field of rationality", is actually possible. Therefore, even if our main result seems abstract, one can admit and use it very easily for some practical applications, albeit forgetting about the proofs.

Let

$$
\dot{x}=X(x)
$$

be an analytic differential equation defined by an analytic vector field $X$ over a complex connected manifold $M$ of complex dimension $m$ ( $m=2 n$ in the symplectic case).

To a non-stationary solution $\phi_{t}\left(x_{0}\right):=\phi\left(x_{0}, t\right)$, we associate an immersion $\iota: \Gamma \rightarrow M$, $\Gamma$ being a connected Riemann surface. Consider the local situation to start with. In this case, we can identify $M$ with an open subset of $\mathbf{C}^{m}$ and $\Gamma$ with an open subset of $\mathbf{C}$, and assume $\iota$ is an embedding. The initial data are $t_{0}=0 \in \Gamma$ and $x_{0}=\left(x_{0,1}, \ldots, x_{0, m}\right) \in \mathbf{C}^{m}$. The coordinate functions of the solution will be denoted as $\phi=\left(\phi_{1}, \ldots, \phi_{m}\right)$. We can assume this solution is maximal with the initial data, as well as defined for initial $y_{0}$ close enough to $x_{0}$.

$4^{\text {e }}$ SÉRIE - TOME $40-2007-\mathrm{N}^{\circ} 6$ 
We consider the germ of the flow $\phi$ along the graph $\Delta=\{(t, \iota(t)) \mid t \in \Gamma\}$ a family of germs of diffeomorphisms $\phi_{t}:\left(\mathbf{C}^{m}, x_{0}\right) \rightarrow\left(\mathbf{C}^{m}, \phi_{t}\left(x_{0}\right)\right)$. Then, we have the convergent power series

$$
\phi_{t}\left(y_{0}\right)=\sum_{k \geqslant 0} \phi_{t}^{(k)}\left(x_{0}\right)\left(y_{0}-x_{0}\right)^{k}
$$

where we introduce $\phi_{t}^{(0)}\left(x_{0}\right):=\phi_{t}\left(x_{0}\right)$ and $\phi_{t}^{(k)}\left(x_{0}\right):=D_{x_{0}}^{k} \phi_{t} / k !$.

Our immediate goal is to obtain the equations for the derivatives of $\phi_{t}$ with respect to $x_{0}$ and, therefore, for $\phi_{t}^{(k)}\left(x_{0}\right)$. Working first in coordinates, let $X_{i}$ be the components of $X$ and let us introduce the notation

$$
D_{i_{1}, \ldots, i_{s}}^{s} \phi_{j}=\frac{\partial^{s} \phi_{j}}{\partial x_{0, i_{1}} \ldots \partial x_{0, i_{s}}}, \quad D_{k_{1}, \ldots, k_{r}}^{r} X_{i}=\frac{\partial^{r} X_{i}}{\partial x_{k_{1}} \ldots \partial x_{k_{r}}} .
$$

It will be also useful to introduce the power series expansion for $X$

$$
X(y)=\sum_{k \geqslant 0} X^{(k)}(x)(y-x)^{k}
$$

with $X^{(0)}(x):=X(x)$, similarly to what was done before. It is clear that $\phi_{t}^{(k)}\left(x_{0}\right)$ and $X^{(k)}(x)$ are $k$-linear symmetric maps.

Then, by successive derivation of (10) with respect to the coordinate functions of $x_{0}$ and exchange of the order of the derivations, we obtain the desired equations

$$
\begin{aligned}
\frac{d}{d t} D_{k} \phi_{j}= & D_{i} X_{j} D_{k} \phi_{i}, \\
\frac{d}{d t} D_{k_{1}, k_{2}}^{2} \phi_{j}= & D_{i} X_{j} D_{k_{1}, k_{2}}^{2} \phi_{i}+D_{i_{1}, i_{2}}^{2} X_{j} D_{k_{1}} \phi_{i_{1}} D_{k_{2}} \phi_{i_{2}}, \\
\frac{d}{d t} D_{k_{1}, k_{2}, k_{3}}^{3} \phi_{j}= & D_{i} X_{j} D_{k_{1}, k_{2}, k_{3}}^{3} \phi_{i}+D_{i_{1}, i_{2}}^{2} X_{j} D_{k_{1}, k_{2}}^{2} \phi_{i_{1}} D_{k_{3}} \phi_{i_{2}} \\
& +D_{i_{1}, i_{2}}^{2} X_{j} D_{k_{1}, k_{3}}^{2} \phi_{i_{1}} D_{k_{2}} \phi_{i_{2}}+D_{i_{1}, i_{2}}^{2} X_{j} D_{k_{1}} \phi_{i_{1}} D_{k_{2}, k_{3}}^{2} \phi_{i_{2}} \\
& +D_{i_{1}, i_{2}, i_{3}}^{3} X_{j} D_{k_{1}} \phi_{i_{1}} D_{k_{2}} \phi_{i_{2}} D_{k_{3}} \phi_{i_{3}}, \\
\ldots= & \ldots,
\end{aligned}
$$

where summation is performed with respect to repeated indices as usual. The first line in (12) gives the first variational equations $V E_{1}$, the first two (resp. three) lines give the second (resp. third) variational equations $V E_{2}$ (resp. $V E_{3}$ ), etc. It is possible to write these equations in a general, more compact form, by making use of $\phi_{t}^{(k)}$ and $X^{(k)}$ :

$$
\begin{aligned}
\dot{\phi}_{t}^{(1)} & =X^{(1)} \phi_{t}^{(1)}, \\
\dot{\phi}_{t}^{(2)} & =X^{(1)} \phi_{t}^{(2)}+X^{(2)}\left(\phi_{t}^{(1)}\right)^{2}, \\
\dot{\phi}_{t}^{(3)} & =X^{(1)} \phi_{t}^{(3)}+2 X^{(2)}\left(\phi_{t}^{(2)}, \phi_{t}^{(1)}\right)+X^{(3)}\left(\phi_{t}^{(1)}\right)^{3}, \\
\ldots & =\ldots,
\end{aligned}
$$

or, in general, 


$$
\dot{\phi}_{t}^{(k)}=\sum \frac{j !}{m_{1} ! \cdots m_{s} !} X^{(j)}\left(\left(\phi_{t}^{\left(i_{1}\right)}\right)^{m_{1}},\left(\phi_{t}^{\left(i_{2}\right)}\right)^{m_{2}}, \ldots,\left(\phi_{t}^{\left(i_{s}\right)}\right)^{m_{s}}\right), \quad k \geqslant 1,
$$

where the $X^{(k)}$ are evaluated at $\phi_{t}\left(x_{0}\right)$, the symmetry of the multilinear maps has been used and the composition of multilinear maps has the obvious meaning: a term in the right hand side of (14) acts on a string of $k$ vectors $\left(u_{1}, \ldots, u_{k}\right)$ as

$$
X^{(j)}\left(\phi_{t}^{\left(i_{1}\right)}\left(u_{1}, \ldots, u_{i_{1}}\right), \ldots, \phi_{t}^{\left(i_{s}\right)}\left(u_{k-i_{s}+1}, \ldots, u_{k}\right)\right) .
$$

In (14) the summations are carried out for

$$
1 \leqslant j \leqslant k, i_{1}>i_{2}>\cdots>i_{s}, \quad \sum_{r=1}^{s} m_{r}=j, \quad \sum_{r=1}^{s} m_{r} i_{r}=k .
$$

The obvious initial conditions for $\phi_{t}^{(k)}\left(x_{0}\right)$ are $\phi_{t}^{(1)}\left(x_{0}\right)=\operatorname{id}$ and $\phi_{t}^{(k)}\left(x_{0}\right)=0$ for $k>1$. The equations for $V E_{1}$ are linear and homogeneous, while the remaining $V E_{k}$ are neither of both, the non-linear part depending on the previous $\phi_{t}^{(j)}, j<k$ as is evident from (12) or (13). The linear homogeneous part has the same form for all of the equations. Hence, as said before, the solutions can be obtained in a recurrent way by quadratures using, for instance, the variation of constants method. More specifically: if we assume that we know a solution $\left(\phi_{t}^{(1)}, \ldots, \phi_{t}^{(k)}\right)$ of $V E_{k}$, then we can write the equation for the new terms appearing in $V E_{k+1}$ :

$$
\dot{\phi}_{t}^{(k+1)}\left(x_{0}\right)=X^{(1)}\left(\phi_{t}\left(x_{0}\right)\right) \phi_{t}^{(k+1)}\left(x_{0}\right)+P\left(\phi_{t}^{(1)}, \ldots, \phi_{t}^{(k)}\right) .
$$

In Equation (15) $P$ denotes polynomial expressions in the components of its arguments. The coefficients depend on $t$ through $X^{(j)}\left(\phi_{t}\left(x_{0}\right)\right)$.

The problem is now to find a system of linear equations for $\left(\phi_{t}^{(1)}\left(x_{0}\right), \ldots, \phi_{t}^{(k)}\left(x_{0}\right)\right)$ equivalent to the system of higher variational equations. It is enough to write the equations satisfied by the monomials appearing in $P$. This is the content of the next lemma, and is similar to typical procedures in automatic differentiation and Taylor integration routines.

Lemma 4. - Let $z \in \mathbf{C}^{q}$. Assume the components $\left(z_{1}, \ldots, z_{q}\right)$ of $z$ satisfy linear homogeneous differential equations $\dot{z}_{i}=\sum_{j=1}^{q} a_{i j}(t) z_{j}$. Then the monomials $z^{k}$ of order $|k|$ also satisfy a system of linear homogeneous differential equations.

Proof. - Let $k=\left(k_{1}, \ldots, k_{q}\right)$ be a multi-index of non-negative integers. Then

$$
\frac{d}{d t} z^{k}=\sum_{j=1}^{q}\left(k_{j} z_{j}^{k_{j}-1} \sum_{r=1}^{q} a_{j r} z_{r} \prod_{i=1, i \neq j}^{q} z_{i}^{k_{i}}\right),
$$

the right hand side being also homogeneous of degree $|k|$ in $z$.

We observe that the above lemma is nothing but the pullback to the symmetric fibre bundle, $S^{k}\left(\mathbf{C}^{q}\right)$, of the connection associated to the linear differential equations.

In our application to linearise the $V E_{k}$ it is clear that the $a_{j r}=a_{j r}(t)$ depend on $t$ through the components of the $X^{(i)}\left(\phi_{t}\left(x_{0}\right)\right)$ for $1 \leqslant i \leqslant k$. We realise that after the last equation corresponding to $V E_{k}$ we can supplement the system of linear differential equations with the 
equations for the components of $\left(\left(\phi_{t}^{\left(i_{1}\right)}\right)^{m_{1}},\left(\phi_{t}^{\left(i_{2}\right)}\right)^{m_{2}}, \ldots,\left(\phi_{t}^{\left(i_{s}\right)}\right)^{m_{s}}\right)$. More concretely, after the first, second, ..., equations in (13) we must add equations of the form

$$
\begin{aligned}
& \frac{d}{d t}\left(\phi_{t}^{(1)}\right)^{2}=L_{2}\left(\phi_{t}^{(1)}\right)^{2}, \\
& \frac{d}{d t}\left(\phi_{t}^{(2)}, \phi_{t}^{(1)}\right)=L_{3,1}\left(\phi_{t}^{(2)}, \phi_{t}^{(1)}\right), \quad \frac{d}{d t}\left(\phi_{t}^{(1)}\right)^{3}=L_{3,2}\left(\phi_{t}^{(1)}\right)^{3},
\end{aligned}
$$

where the coefficients in $L_{i, j}$ are obtained using (16). In this way we obtain recurrently the desired linearised version $L V E_{k}$.

It is easy to reformulate the construction of this linearisation in matrix form using a composition by an arbitrary scalar function (or jet) at the target.

At this point we need to consider the action of the flow on jets of functions. We set $z=$ $\phi_{t}\left(y_{0}\right)-\phi_{t}\left(x_{0}\right)$. Let $f:\left(M, \phi_{t}\left(x_{0}\right)\right) \rightarrow(\mathbf{C}, 0)$ be a germ of holomorphic function. Let us write its power series expansion:

$$
f(z)=f^{(1)} z+f^{(2)}(z)^{2}+\cdots .
$$

We will assume that, for each $k \in \mathbf{N}^{*}$, the multilinear symmetric map $f^{(k)}$ is independent of $t$. Beware of the fact that the source of the infinite jet of $f$ depends on $t$ : among the natural coordinates of this jet, only the source coordinates $\phi_{t}\left(x_{0}\right)$ depend on $t$. Then $f \mapsto f \circ \phi_{t}$ is a linear map. We set $f \circ \phi_{t}=\varphi_{t}\left(f^{(1)}, f^{(2)}, \ldots\right)$. Again $\left(f^{(1)}, f^{(2)}, \ldots\right) \mapsto \varphi_{t}\left(f^{(1)}, f^{(2)}, \ldots\right)$ is a linear map.

For each fixed value of $\left(f^{(1)}, f^{(2)}, \ldots\right)$, independently of $t$, we can write a differential system, of infinite order, satisfied by $\varphi_{t}\left(f^{(1)}, f^{(2)}, \ldots\right)$. Then $\varphi_{t}$ will appear as a fundamental solution of this linear system. Using "infinite matrix" form, we will obtain a linearisation $L V E_{\omega}$ of the higher variational equations altogether. Finally we will obtain, for each $k$, the linearisation $L V E_{k}$ of $V E_{k}$ by truncation, i.e., replacing $\left(f^{(1)}, f^{(2)}, \ldots\right)$ by the $k$-jet $\left(f^{(1)}, f^{(2)}, \ldots, f^{(k)}\right)$.

Then $\left(f^{(1)}, f^{(2)}, \ldots\right) \mapsto \varphi_{t}\left(f^{(1)}, f^{(2)}, \ldots\right)$ yields

$$
F \mapsto F \Phi_{t}
$$

in matrix form, where $F$ is the infinite vector containing all components of the $f^{(k)}$, supplemented at every order by the required products of lower order terms. We see that $\varphi_{t} \rightarrow \Phi_{t}$ is a faithful representation of $\operatorname{Diff}\left(\mathbf{C}^{m}, 0\right)$ in a group of invertible infinite-dimensional matrices.

We have $\frac{d}{d t}\left(f \circ \phi_{t}\right)=d f(X) \circ \phi_{t}$. In matrix form, the infinite matrix $\Phi_{t}$ satisfies a linear differential system

$$
\dot{\Phi}_{t}=A_{t} \Phi_{t},
$$

where the infinite matrix $A_{t}$ collects all coefficients appearing in (12) and (17).

The $L V E_{k}$ are obtained by truncation of triangular matrices:

$$
j^{\dot{k}} \Phi=j^{k} A j^{k} \Phi
$$

The map $j^{k} \varphi \rightarrow j^{k} \Phi$ is a faithful representation of $\operatorname{Diff}^{k}\left(\mathbf{C}^{m}, 0\right)$ in a group of invertible upper triangular matrices. It follows that $j^{k} A$ takes its value in a corresponding Lie algebra, in turn a faithful representation of $J^{k}\left(\mathbf{C}^{m}, 0\right)$. 
PROPOSITION 8. - The $V E_{k}$ and the $L V E_{k}$ are equivalent. More precisely, we can establish a bijection between solutions $t \mapsto \phi_{t}$ of the $V E_{k}$ such that $\phi_{0}=\mathrm{id}$ and fundamental solutions of the $L V E_{k}$ equal to the identity for $t=0$.

Proof. - It follows immediately from the preceding considerations.

We now have the following problem: preceding computations rely on a coordinate choice, but in order to apply differential Galois theory we need a global geometric formulation in terms of connections. We will now give such a geometric intrinsic formulation. The fundamental idea relies on the use of duality. The starting point is to replace the family of diffeomorphisms $\phi_{t}:\left(\mathbf{C}^{m}, x_{0}\right) \rightarrow\left(\mathbf{C}^{m}, \phi_{t}\left(x_{0}\right)\right)$ by the family of inverse diffeomorphisms $\psi_{t}=\phi_{t}^{-1}:\left(\mathbf{C}^{m}, \phi_{t}\left(x_{0}\right)\right) \rightarrow\left(\mathbf{C}^{m}, x_{0}\right)$. The key point is that now the targets are independent of $t$. We have $\phi_{0}=\mathrm{id}, \psi_{0}=\mathrm{id}$.

We denote by $f$ a germ of holomorphic function at $x_{0}$ on $M$, vanishing at $x_{0}$. The map $f \mapsto f \circ \psi_{t}$ is linear. We write it in matrix form

$$
F \mapsto F \Psi_{t} .
$$

We clearly have $\Psi_{0}=\Phi_{0}=I$ and $\Phi_{t} \Psi_{t}=I$. By derivation, we obtain

$$
\dot{\Psi}_{t}=-\Phi_{t}^{-1} \dot{\Phi}_{t} \Phi_{t}^{-1}
$$

and, using (19):

$$
\dot{\Psi}_{t}=-\Psi_{t} A_{t}
$$

and by transposition

$$
{ }^{t} \dot{\Psi}_{t}=\left(-{ }^{t} A_{t}\right)^{t} \Psi_{t} .
$$

Therefore ${ }^{t} \Psi_{t}$ is the "fundamental solution" of the "dual" differential system of (19). By truncation we have a true duality: $j^{k}\left({ }^{t} \Psi_{t}\right)$ is the fundamental solution of the dual system of $j^{\dot{k}} \Phi_{t}=j^{k} A_{t} j^{k} \Phi_{t}$. We denote this dual system by $L V E_{k}^{*}$.

Now we will see these dual systems are associated to natural connections on fibre bundles admitting the groups $\operatorname{Diff}^{k}\left(\mathbf{C}^{m}, 0\right)$ as structure groups. A connection on a fibre bundle is essentially a parallel transport on the fibres and such a transport will happen to appear in our situation in a natural way.

Let $M$ be a connected complex manifold of dimension $m$. Let $X$ be a holomorphic vector field on $M$. We choose a non-stationary solution $\iota: \Gamma \rightarrow M, \Gamma$ being a connected Riemann surface and $\iota$ an embedding. Let $\Delta \in \Gamma \times M$ be the graph of $\iota$.

Using $\Delta$, we can interpret the collection of germs of analytic manifold $\{(M, \xi) \mid \xi=$ $\iota(\tau), \tau \in \Gamma\}$ along $\iota(\Gamma)$ as a non-linear locally trivial fibre bundle on $\Gamma$, the fibres being isomorphic to $\left(\mathbf{C}^{m}, 0\right)$ and the structure group being $\operatorname{Diff}\left(\mathbf{C}^{m}, 0\right)$. Then we can regard the flow of (10) along $\iota(\Gamma)$ as a parallel transport along this fibre bundle. Now, if we replace the collection of germs of the analytic manifold $\{(M, \xi) \mid \xi=\iota(\tau), \tau \in \Gamma\}$ by the "dual" collection of germs of holomorphic functions $\left\{\mathcal{O}_{\xi} \mid \xi=\iota(\tau), \tau \in \Gamma\right\}$, we obtain a locally trivial infinite-dimensional holomorphic linear fibre bundle $J_{\Gamma}^{\omega} M=\iota^{*} J^{\omega} M$ on $\Gamma$, the fibres being isomorphic to the complex vector spaces $\left(\mathbf{C}\left\{x_{1}, \ldots, x_{m}\right\}, 0\right)$ and the structure group being $\operatorname{Diff}\left(\mathbf{C}^{m}, 0\right)$. The flow acting "dually" on the functions gives a linear parallel transport on this bundle corresponding to a "connection" $\nabla_{\omega}$. Replacing the spaces $\mathcal{O}_{\xi}$ of germs of holomorphic functions by the quotient spaces of $k$-jets $J^{k}(M, \xi)$, we obtain locally trivial finitedimensional holomorphic linear fibre bundles on $\Gamma: \iota^{*} J^{k} M=J_{\Gamma}^{k} M$. By quotients of our parallel 
transport we have holomorphic parallel transports on the bundles $J_{\Gamma}^{k} M$. These parallel transports define holomorphic connections $\nabla_{k}$ which are quotients of $\nabla_{\omega}$. We will see that $\left(J_{\Gamma}^{k} M, \nabla_{k}\right)$ corresponds to $L V E_{k}^{*}$ if we introduce local coordinates.

It is possible to justify the preceding considerations using Grothendieck's definition of infinitesimal calculus [32] (infinitesimal neighbourhoods of the diagonal). Here we shall do the work explicitly and in an elementary way.

We will build each connection locally and subsequently check that our constructions glue together yielding global connections. Hence we can assume $\Gamma$ is a simply connected open subset of C. For each non-vanishing holomorphic vector field $\delta$, we will then define the covariant derivative $\nabla_{\delta}$ associated to $\nabla$. It is sufficient to do that for the vector field $\delta=\frac{d}{d t}, t$ being a local coordinate.

We introduce the vector field $\tilde{X}=\frac{d}{d t}+X$ on $\Gamma \times M$. The graph $\Delta$ is invariant by $\tilde{X}$. By definition the horizontal sections of $\nabla_{\omega}$ are the first integrals of $\widetilde{X}$. More precisely,

Definition 2.- Let $t_{0}, t_{1} \in \Gamma$ and let $f_{0}, f_{1}$ be two germs of holomorphic functions on $M$, respectively at the points $\xi_{0}=\iota\left(t_{0}\right)$ and $\xi_{1}=\iota\left(t_{1}\right)$. We will say that we obtain $f_{1}$ from $f_{0}$ by parallel transport (from $t_{0}$ to $t_{1}$ ), if there exists a first integral $f:(\Gamma \times M, \Delta) \rightarrow \mathbf{C}$ of $\widetilde{X}$ holomorphic on an open neighbourhood of $\Delta$ in $\Gamma \times M$ such that $f\left(t_{0}, \xi\right)=f_{0}(\xi)$ and $f\left(t_{1}, \xi\right)=f_{1}(\xi)$. In this definition we allow restriction to $\Gamma$, this open set remaining simply connected and $t_{0}, t_{1}$ remaining fixed.

The function $f$ is a first integral of $\widetilde{X}$ if and only if

$$
L_{\widetilde{X}} f(t, \xi)=L_{d / d t} f(t, \xi)+L_{X} f(t, \xi)=0 .
$$

This is equivalent to the following condition:

$$
\dot{f}(t, \xi):=\frac{\partial}{\partial t} f(t, \xi)=-\frac{\partial}{\partial \xi} f(t, \xi) X(\xi) .
$$

LEMmA 5. - Let $f:(\Gamma \times M, \Delta) \rightarrow \mathbf{C}$ be a holomorphic function on a neighbourhood of $\Delta$ in $\Gamma \times M$. Let $t_{0} \in \Gamma$ be a fixed point. The following conditions are equivalent:

(i) $f_{t}$ (where $\left.f_{t}(\xi)\right)=f(t, \xi)$ ) comes by parallel transport from $f_{t_{0}}$ for every $t \in \Gamma$.

(ii) There exists a unique family of germs of holomorphic diffeomorphisms $\phi_{t}:\left(M, \iota\left(t_{0}\right)\right)$ $\rightarrow(M, \iota(t))$ such that $f_{t} \circ \phi_{t}=f_{t_{0}}$, for $t \in \Gamma$.

Moreover, if these conditions are satisfied, then:

(a) $\phi_{t_{0}}(\xi)=\phi\left(t_{0}, \xi\right)=\xi\left(\right.$ i.e., $\left.\phi_{t_{0}}=\mathrm{id}\right)$;

(b) $\dot{\phi}_{t}(\xi)=\frac{\partial}{\partial t} \phi(t, \xi)=X(\phi(t, \xi))$, that is $\phi$ is the flow of the field $X$, with initial conditions $\left(t_{0}, \xi_{0}\right)$.

Proof. - The proof is clear: conditions (i) and (ii) are obviously equivalent to the condition that $f(t, x)$ is constant along the flow curves. Later on we will need a similar lemma for $k$-jets. Hence, we give another proof that will be later useful in the jet context.

We assume that (ii) is satisfied: $f_{t} \circ \phi_{t}=f_{t_{0}}$. Then, by $t$-derivation we have:

$$
\dot{f}(t, \phi(t, \xi))+D_{x} f(t, \phi(t, \xi)) \dot{\phi}(t, \xi)=\dot{f}(t, \phi(t, \xi))+D_{x} f(t, \phi(\xi)) X(\phi(\xi))=0 .
$$

We conclude using equivalence of (i) and (22).

Let now $j^{k} f_{t}(\xi)=j^{k} f(t, \xi)$ be a holomorphic family of $k$-jets along $\Delta$ in $\Gamma \times M$ (a holomorphic function on the $k$-infinitesimal neighbourhood $\Delta^{(k)}$ of $\Delta$ in $\Gamma \times M$ ). For a 
fixed $t$ consider $j^{k} f_{t} \in J^{k}(M, \iota(t))$. We will say that it is a first integral of $\widetilde{X}$ if and only if

$$
\left(j^{k} f\right)^{\cdot}(t, \xi)=\frac{\partial}{\partial t} j^{k} f(t, \xi)=-\frac{\partial}{\partial \xi} j^{k} f(t, \xi) X(\xi)
$$

in the evident jet sense. If we interpret $j^{k} f$ as a holomorphic section of $J_{\Gamma}^{k} M$, (24) is a holomorphic differential system

$$
\dot{Y}=j^{k} A_{t} Y \text {. }
$$

The matrix function $t \mapsto A_{t}$ takes its value in $\mathcal{L}^{k}\left(\mathbf{C}^{m}, 0\right)$. Therefore (25) corresponds to a connection with structure group $\operatorname{Diff}^{k}\left(\mathbf{C}^{m}, 0\right)$. Moreover, if we are in a symplectic situation ( $M$ symplectic, $m=2 n, X$ Hamiltonian), then $A_{t} \in \mathcal{L}_{s p}^{k}\left(\mathbf{C}^{2 n}, 0\right)$ and the structure group is $\operatorname{Difff}_{S p}^{k}\left(\mathbf{C}^{2 n}, 0\right)$.

We can now give an analogue of Lemma 5 for $k$-jets.

LEMMA 6. - Let $j^{k} f_{t}(\xi)=j^{k} f(t, \xi)$ be a holomorphic family of $k$-jets along $\Delta$ in $\Gamma \times M$. Let $t_{0} \in \Gamma$ be a fixed point. The following conditions are equivalent:

(i) $j^{k} f(t, \xi)$ is a first integral of $\widetilde{X}$ (in the $k$-jet sense).

(ii) There exists a unique family of germs of $k$-jets of holomorphic diffeomorphisms $j^{k} \phi_{t}:\left(M, \iota\left(t_{0}\right)\right) \rightarrow(M, \iota(t))$ such that $j^{k} f_{t} \circ \phi_{t}=j^{k} f_{t_{0}}$, for $t \in \Gamma$.

Moreover, if these conditions are satisfied, then:

(a) $j^{k} \phi_{t_{0}}=j^{k}$ id.

(b) $\left(j^{k} \phi\right)^{\cdot}(t, \xi)=\frac{\partial}{\partial t} j^{k} \phi(t, \xi)=j^{k}(X(\phi(t, \xi)))$.

Proof. - We prove the implication (ii) $\Rightarrow$ (i) by $t$-derivations in the functional case above. Let us now prove the implication (i) $\Rightarrow$ (ii).

Assume we have (24):

$$
\left(j^{k} f\right) \cdot(t, \xi)=\frac{\partial}{\partial t} j^{k} f(t, \xi)=-\frac{\partial}{\partial \xi} f(t, \xi) X(\xi) .
$$

We interpret this equation as a differential system (25)

$$
\dot{Y}=j^{k} A_{t} Y
$$

where $j^{k} A_{t} \in \mathcal{L}^{k}\left(\mathbf{C}^{m}, 0\right)$ (resp. $j^{k} A_{t} \in \mathcal{L}_{s p}^{k}\left(\mathbf{C}^{m}, 0\right)$ in the symplectic case). Let $j^{k} \Phi_{t}$ be the unique holomorphic fundamental solution of this system such that $j^{k} \Phi_{0}=\mathrm{id}$. Then we have the following result.

Lemma 7. - We have $j^{k} \Phi_{t} \in \operatorname{Diff}^{k}\left(\mathbf{C}^{m}, 0\right)$ (resp. $j^{k} \Phi_{t} \in \operatorname{Diff}_{S p}^{k}\left(\mathbf{C}^{2 n}, 0\right)$ in the symplectic case).

This follows from the following Lemma [24] (6.25 Lemme, p. 238).

LEMMA 8. - Let $G$ be a linear complex algebraic group and $\mathcal{G}$ its Lie algebra. Let $\dot{Y}=A_{t} Y$ be a holomorphic linear system in a neighbourhood of $t=0$. We assume that $A_{t} \in \mathcal{G}$. Then the unique holomorphic fundamental solution of this system takes its value in $G$ (more precisely in the identity component $G^{0}$ of $G$ ).

Now it is easy to end the proof of Lemma 6.

$4^{\text {e }}$ SÉRIE - TOME $40-2007-\mathrm{N}^{\circ} 6$ 


\section{The differential Galois groups of the variational equations. The main theorem}

Let

$$
\dot{x}=X_{H}(x)
$$

be the analytic differential equation defined by a Hamiltonian vector field $X_{H}$ over a complex connected symplectic manifold $M$ of complex dimension $2 n$. To a non-stationary solution we associate an immersion $\iota: \Gamma \rightarrow M, \Gamma$ being a connected Riemann surface. We choose a nontrivial derivation $\partial$ over the field $k_{\Gamma}$ of meromorphic functions on $\Gamma$.

Let $m \in \Gamma$. We denote by $\mathcal{O}_{m}$ (resp. $\mathcal{M}_{m}$ ) the algebra of germs of holomorphic functions over $\Gamma$ at $m$ (resp. the field of germs of meromorphic functions over $\Gamma$ at $m$ ). Here we will use the linear variational equations $L V E_{k}$ of order $k$, which we defined in the preceding section. They are holomorphic connections $\nabla_{k}$ over the "restrictions" (more precisely pullbacks by $\left.\iota\right)\left(J^{k}\right)_{\Gamma}^{*} M$ of the dual bundles $\left(J^{k}\right)^{*} M$ of the fibre bundles $J_{M}^{k}$ of $k$-jets of scalar holomorphic functions on $\Gamma$. The structure groups of these connections are the groups of symplectic $k$-jets of diffeomorphisms $\operatorname{Diff}_{S p}^{k}\left(\mathbf{C}^{2 n}, 0\right)$. We will also use the $L V E_{k}^{*}$ which are holomorphic connections $\nabla_{k}^{*}$ on the bundles $J_{\Gamma}^{k} M$. The structure groups of these connections are also the groups of symplectic $k$-jets of diffeomorphisms Diff ${ }_{S p}^{k}\left(\mathbf{C}^{2 n}, 0\right)$. For $k=1$ we get $\left(J^{1}\right)_{\Gamma}^{*} M=T_{\Gamma} M$ and $J_{\Gamma}^{1} M=T_{\Gamma}^{*} M$ and the structure group is $S p\left(\mathbf{C}^{2 n}, 0\right)$.

We recall that, for $r \leqslant k,\left(J^{r}\right)_{\Gamma}^{*} M$ (resp. $\left.J_{\Gamma}^{r} M\right)$ is a sub-bundle (resp. a quotient bundle) of $\left(J^{k}\right)_{\Gamma}^{*} M$ and that $\nabla_{r}$ (resp. $\left.\nabla_{r}^{*}\right)$ is a subconnection (resp. a quotient) of $\nabla_{k}$ (resp. $\nabla_{k}^{*}$ ).

We recall also (cf. Appendix A) that each holomorphic bundle $J_{\Gamma}^{k} M$ or $\left(J^{k}\right)_{\Gamma}^{*} M$ is meromorphically trivialisable over $\Gamma$ (as a bundle with structure group $\operatorname{Diff}_{S p}^{k}\left(\mathbf{C}^{2 n}, 0\right)$ ) and we will assume in this section that we have fixed such a trivialisation for each $k$. Then we can write $L V E_{k}$ or $L V E_{k}^{*}$ as a differential system of order one:

$$
\dot{Y}=A_{k} Y
$$

where $A_{k}$ is a meromorphic function taking its values in the Lie algebra $\mathcal{L}_{s p}^{k}\left(\mathbf{C}^{2 n}, 0\right)$.

This differential system can have singularities, depending on the choice of trivialisation; however they are clearly removable singularities. In the following we will work at a regular point for the most part, but the results extend immediately to a removable singularity.

Let $m \in \Gamma$. Applying Cauchy's theorem, we obtain a fundamental system of solutions $F_{k}$ whose entries belong to $\mathcal{M}_{m}$ (and the same happens for $F_{k}^{*}$ ) for the trivialisations of the $L V E_{k}$ (and $L V E_{k}^{*}$ ). They are holomorphic, that is $\in \mathcal{O}_{m}$ if $m$ is regular, and meromorphic if $m$ is an apparent singularity. We denote by $L_{k}$ the sub-differential field of $\mathcal{M}_{m}$ generated by the entries of $F_{k}$ over $k_{\Gamma}$. It is also the sub-differential field of $\mathcal{M}_{m}$ generated by the entries of $F_{k}^{*}$ over $k_{\Gamma}$ and it is independent of the choice of the trivialisation. Then $L_{k}$ is a Picard-Vessiot extension of $k_{\Gamma}$ associated to $L V E_{k}$ and also to $L V E_{k}^{*}$. We have inclusions of differential fields $k_{\Gamma} \subset L_{1} \subset \cdots \subset L_{k} \subset L_{k+1} \subset \cdots$. All the corresponding extensions of differential fields are normal.

By definition "the" differential Galois group Gal $\nabla_{k}$ of $L V E_{k}$ (or $L V E_{k}^{*}$ ) is Gal $\nabla_{k}=$ Aut $_{k_{\Gamma}}^{\partial} L_{k}$. It depends up to a non-natural isomorphism on the choice of a Picard-Vessiot extension, and thus, in this case, on the choice of $m$.

Using the differential Galois correspondence, we have short exact sequences

$$
\{e\} \rightarrow \operatorname{Aut}_{L_{k}}^{\partial} L_{k+1} \rightarrow \operatorname{Gal} \nabla_{k+1} \rightarrow \operatorname{Gal} \nabla_{k} \rightarrow\{e\} .
$$


We denote by $\mathrm{Sol}_{k}\left(\right.$ resp. $\mathrm{Sol}_{k}^{*}$ ) the linear complex space generated by the entries of $F_{k}$ (resp. $F_{k}^{*}$ ). From each of these spaces we easily obtain the solutions of the (non-linear) classical $k$-variational equation $V E_{k}$.

The differential Galois group Gal $\nabla_{k}=\operatorname{Aut}_{k_{\Gamma}}^{\partial} L_{k}$ acts naturally on the linear spaces $\mathrm{Sol}_{k}$ and $\mathrm{Sol}_{k}^{*}$ and we obtain natural faithful representations of $\mathrm{Gal} \nabla_{k}$ in $\mathrm{GL}\left(\mathrm{Sol}_{k}\right)$ and $\mathrm{GL}\left(\mathrm{Sol}_{k}^{*}\right)$. We will use these representations to build a natural homomorphism of algebraic groups

$$
\operatorname{Gal} \nabla_{K} \rightarrow \operatorname{Diff}_{S p}^{k}(M, m)
$$

We denote $\operatorname{Diff}_{S p}^{k}(M, m)$ the pullback of $\operatorname{Diff}_{S p}^{k}(M, \iota(m))$ by the map $\iota$. We will see that this homomorphism is injective. By definition its image will be "the" differential Galois group of the higher variational equation $V E_{k}$.

Our construction will be local. We will use coordinates, although the result is independent of any choice thereof as one easily checks. Near $\iota(m)$ we can choose Darboux coordinates on the symplectic manifold $M$ centred at $\iota(m)$. Then we can use these coordinates to locally trivialise our scalar jet bundles $\left(J^{k}\right)_{\Gamma}^{*} M$ and $J_{\Gamma}^{k} M$ : we write them as products of the basis by $J^{k}\left(\mathbf{C}^{2 n}, 0\right)$ and $\left(J^{k}\right)^{*}\left(\mathbf{C}^{2 n}, 0\right)$, using the standard coordinates on the jets-we identify $(M, \iota(m))$ with $\left(\mathbf{C}^{2 n}, 0\right)$ with its standard symplectic structure of linear space. Then we can interpret an element $P$ of the fibre at $\iota(m)$ of $J_{M}^{k}$ as an element of $J^{k}\left(\mathbf{C}^{2 n}, 0\right)$ and this last element as a polynomial in $y$ with values in C. On $\Gamma$ we can use the temporal parametrisation $t$ setting $t=0$ at $m=x_{0}$.

We will make an essential use of the following result [62, Theorem 14, Appendix C, page 92].

Proposition 9. - Let $G$ be a complex linear algebraic group. Let $\nabla$ be a $G$-meromorphic connection on a trivial $G$-bundle over a connected Riemann surface $\Gamma$. Then its differential Galois group "is" a Zariski closed subgroup of G.

As before, we denote by $\phi_{t}$ the flow map ( $\phi_{0}=$ id) and we set $\psi_{t}=\phi_{t}^{-1} ; \psi_{t}^{k}$ is the $k$-jet of $\psi_{t}$. Its source is $\phi\left(x_{0}\right)$ and its target is $x_{0}=m$. Let $f \in J^{k}\left(\mathbf{C}^{2 n}, 0\right)$. We consider it as an initial condition for $L V E_{k}^{*}$. The corresponding solution is (locally) $f(t)=f \circ \psi_{t}^{k}(f(0)=f)$. We have $f(t) \in \mathrm{Sol}_{k}^{*}$ and $\sigma \in \mathrm{Gal}_{k}$ transforms $f(t)$ into another solution $g(t) \in \mathrm{Sol}_{k}^{*}$. We set $g(0)=g$. Then $g(t)=g \circ \psi_{t}^{k}$. We obtain a faithful representation of Gal $\nabla_{k}=\mathrm{Aut}_{k_{\Gamma}}^{\partial} L_{k}$ in the linear space $J^{k}\left(\mathbf{C}^{2 n}, 0\right)$ (the differential Galois group acts on the initial conditions):

$$
\rho_{k}: \operatorname{Gal} \nabla_{k} \rightarrow \operatorname{GL}\left(J^{k}\left(\mathbf{C}^{2 n}, 0\right)\right), \quad \sigma \mapsto(f \mapsto g) .
$$

This action is clearly compatible with the ordinary product of scalar jets; therefore, if $\sigma \in$ Gal $\nabla_{k}, \rho_{k}(\sigma)$ is an automorphism of the $\mathbf{C}$-algebra $J^{k}\left(\mathbf{C}^{2 n}, 0\right)$. Using Proposition 3, we can interpret $\rho_{k}(\sigma)$ as an element of $J_{0,0}^{k}\left(\mathbf{C}^{2 n}\right)=\operatorname{Diff}^{k}\left(\mathbf{C}^{2 n}, 0\right)$. We obtain a faithful representation (with the same notation):

$$
\rho_{k}: \operatorname{Gal} \nabla_{k} \rightarrow \operatorname{Diff}^{k}\left(\mathbf{C}^{2 n}, 0\right)
$$

It remains to prove that $\rho_{k}(\sigma) \in \operatorname{Diff}^{k}\left(\mathbf{C}^{2 n}, 0\right)$ is a symplectic jet. This is the delicate point. We recall that we choose our trivialisation of $L V E_{k}^{*}$ without changing the structure group $\operatorname{Diff}_{S p}^{k}\left(\mathbf{C}^{2 n}, 0\right)$ : the matrix $A$ of the corresponding differential system takes its values in the Lie algebra $\mathcal{L}_{s p}^{k}\left(\mathbf{C}^{2 n}, 0\right)$. Now we can apply Proposition 9: the image of $\rho_{k}$ in $\operatorname{Diff}^{k}\left(\mathbf{C}^{2 n}, 0\right)$ is contained in the algebraic subgroup $\operatorname{Diff}_{S p}^{k}\left(\mathbf{C}^{2 n}, 0\right)$ (the structure group of our system). We obtain a faithful representation and henceforth indulge in the corresponding abuse of notation:

$$
\rho_{k}: \operatorname{Gal} \nabla_{k} \rightarrow \operatorname{Diff}_{S p}^{k}\left(\mathbf{C}^{2 n}, 0\right) .
$$

$4^{\text {e }}$ SÉRIE - TOME $40-2007-\mathrm{N}^{\circ} 6$ 
It is easy to check that this representation is independent of the choice of trivialisation and local coordinates (it depends only on the point $m$ ) and we obtain a faithful natural representation, without changing notation:

$$
\rho_{k}: \operatorname{Gal} \nabla_{k} \rightarrow \operatorname{Diff}_{S p}^{k}(M, m) .
$$

By definition the image of the homomorphism $\rho_{k}$ in $\operatorname{Diff}_{S p}^{k}(M, m)$ is "the" differential Galois group of the higher variational equation $V E_{k}$. We will denote it $G_{k}$, or $G_{m, k}$ if necessary. If $\gamma$ is a continuous path from $m_{1}$ to $m_{2}$ on $\Gamma$, then the flow from $m_{1}$ to $m_{2}$ along $\gamma$, or more precisely its $k$-jet, induces an isomorphism between $G_{m_{1}, k}$ and $G_{m_{2}, k}$.

PROPOSITION 10. - The natural homomorphism of algebraic groups Gal $\nabla_{k} \rightarrow$ $\operatorname{Diff}_{S p}^{k}(M, m)$ is a morphism of algebraic groups and an injection. Therefore Gal $\nabla_{k} \rightarrow G_{k}$ is an isomorphism of algebraic groups. In other words, the differential Galois groups of the $L V E_{k}$ and of the $V E_{k}$ are isomorphic.

We have commutative diagrams $\left(G \nabla_{k}=\right.$ Gal $\left.\nabla_{k}\right)$

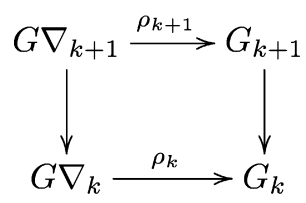

As we saw above (see (28)), the natural maps $G \nabla_{k+1} \rightarrow G \nabla_{k}$ are surjective morphisms of algebraic groups. Therefore the natural maps $G_{k+1} \rightarrow G_{k}$ are also surjective homomorphisms of algebraic groups. This is an essential result in our approach.

Proposition 11. - The natural maps $G_{k+1} \rightarrow G_{k}$ are surjective homomorphisms of algebraic groups.

We denote by $\mathcal{G}_{k}$ the Lie algebra of $G_{k}$. It is identified with a Lie subalgebra of $\mathcal{L}_{s p}^{k}(M, m)$.

By definition the formal differential group of the Hamiltonian system (1) along $\iota(\Gamma)$ is the pro-algebraic group $\hat{G}=\lim _{\leftarrow} G_{k} \subset \operatorname{Diff}^{\infty}(M, m)$.

The Lie algebra $\hat{\mathcal{G}}$ of $\hat{G}$ is $\hat{\mathcal{G}}=\lim _{\leftarrow} \mathcal{G}^{k}$. It is identified with a Lie subalgebra of the Lie algebra of formal Hamiltonian vector fields $\hat{\mathcal{L}}_{s p}^{k}(M, m)$. Then we obtain

PROPOSITION 12. -

(i) The natural maps $\mathcal{G}^{k+1} \rightarrow \mathcal{G}^{k}$ are surjective homomorphisms of Lie algebras.

(ii) The natural maps $\hat{\mathcal{G}} \rightarrow \mathcal{G}^{k}$ are surjective homomorphisms of Lie algebras.

(iii) The natural maps $\hat{G} \rightarrow G_{k}$ are surjective homomorphisms of algebraic groups.

Remark. $-\hat{G}$ (resp. $G_{k}, k \geqslant 1$ ) is Zariski connected if and only if $G_{1}$, that is, the differential Galois group of the $V E_{1}$, is Zariski connected: the successive extensions $G_{k+1} \rightarrow G_{k}(k \geqslant 1)$ are extensions by unipotent algebraic groups. This follows from the recursive integration of the $V E_{k}(k \geqslant 2)$ by the variation of constants method, or if one prefers from the structure of the groups Diff ${ }^{k}$.

The main result of this paper is the following.

THEOREM 5 (Main theorem). - If the Hamiltonian system (1) is completely integrable with meromorphic first integrals along $\iota(\Gamma)$, then

(i) the identity component $\hat{G}^{0}$ of the formal differential Galois group along $\iota(\Gamma)$ is commutative, 
(ii) the identity components $\left(G_{k}\right)^{0}$ of the differential Galois groups of the variational equations along $\iota(\Gamma)$ are commutative $\left(k \in \mathbf{N}^{*}\right)$.

Now, as in the case of the first variational equation, we can add some equilibrium points, or some points at infinity, to $\Gamma$. If we denote this completed Riemann surface by $\bar{\Gamma}$, substitute in the a priori smaller field $k_{\bar{\Gamma}}$ for the differential field $k_{\Gamma}$ and replace $\operatorname{Aut}_{k_{\Gamma}}^{\partial} L_{k}$ by the a priori bigger differential Galois group $\mathrm{Aut}_{k_{\bar{\Gamma}}}^{\partial} L_{k}$, and if we add only equilibrium points or singular points of the field, we have a similar statement, mutatis mutandis. If we add some points at infinity, we need to be careful: if the extended connection is not regular singular at the corresponding points-a property that can be checked on the first variational equation - it is necessary to assume, as in the first variation case, that the first integrals used in the definition of integrability are meromorphic at infinity.

We can also obtain a similar result by considering the local Galois group at a singular point, $a \in \bar{\Gamma}$, i.e. we can assume the field of coefficients to be the differential field $\mathcal{M}_{a}$. Then the non-commutativity of the identity component of the local Galois gives us an obstruction to the meromorphic integrability of the Hamiltonian system in a neighbourhood of the point $\iota(a)$ in $M$. Of course, the most interesting case appears when $a$ is an irregular singular point. This is potentially relevant for the applications, when our complex Hamiltonian system is the complexification of a real system: if $a$ belongs to the real part of $M$, then a local obstruction gives an obstruction to the real integrability (by real meromorphic first integrals).

In order to prove the main theorem, we need a preliminary result. Let $m \in \bar{\Gamma}$ be a nonsingular point $(m \in \Gamma)$. We recall that the differential Galois group $\hat{G}$ of the Hamiltonian system along $\iota(\bar{\Gamma})$, with base point $m$, is a subgroup of $\operatorname{Diff}_{S p}^{\infty}(M, \iota(m))$. If $f_{m}$ is a germ at $\iota(m)$ of meromorphic function on $M$, for $\sigma \in \hat{G}$, we set $f_{m} \circ \sigma=f_{m}^{\sigma}$; it is in general a formal meromorphic germ.

Proposition 13. - We allow for equilibrium points and points at infinity. If $f$ is a meromorphic first integral, and assuming $f$ meromorphic at whichever points at infinity there might be, then

(i) its germ $f_{m}$ at $m \in \Gamma$ is invariant (in the evident sense) by the formal differential Galois group $\hat{G}$ with $m$ as a base point,

(ii) the germ $f_{m}$ is orthogonal to the Lie algebra $\hat{\mathcal{G}}$.

Here orthogonality means, by definition, that $L_{\hat{X}} f_{m}=d f_{m}(\hat{X})=0$ for every formal vector field $\hat{X}$ belonging to $\hat{\mathcal{G}}$.

Proof. - Claim (ii) follows easily from (i), similarly to the proof given in [62] for the first-order variational equations.

It remains to prove statement (i), which seems rather natural and is actually easy to establish if $\Gamma=\bar{\Gamma}$, as we will see, i.e. if there are neither equilibrium points nor points at infinity. This case is sufficient for the applications in this paper-and, for the moment, for all the applications of our main theorem by various authors. We need some preliminary lemmae.

LEMMA 9. - We allow for stationary points and points at infinity. Let $m$ be a point of $\Gamma$. Let $f_{m}$ be a germ of meromorphic function on $(M, m)$ (i.e. on $M$ at $\iota(m)$ ). Let $\hat{H} \subset \hat{G}$ be a Zariskidense subgroup of the differential Galois group $\hat{G} \subset \operatorname{Diff}_{S p}^{\infty}(M, m)$. If $f$ is invariant by $\hat{H}$, then it is invariant by $\hat{G}$.

Proof. - Let $f_{m}=g_{m} / h_{m}$, where $g_{m}, h_{m}$ are germs at $m$ of holomorphic functions on $(M, m)$. If $\sigma \in \hat{H}$, then $f_{m}^{\sigma}=f_{m}$. Therefore $g_{m}^{\sigma} h_{m}-g_{m} h_{m}^{\sigma}=0$ and, setting $J^{k} \sigma=\sigma^{k}$,

$$
J^{k}\left(g_{m}^{\sigma} h_{m}-g_{m} h_{m}^{\sigma}\right)=J^{k} g_{m}^{\sigma^{k}} J^{k} h_{m}-J^{k} g_{m} J^{k} h_{m}^{\sigma^{k}}=0
$$

$4^{e}$ SÉRIE - TOME $40-2007-\mathrm{N}^{\circ} 6$ 
in the $k$-jet sense, for every $k \in \mathbf{N}^{*}$. Then

$$
J^{k} g_{m}^{\sigma^{k}} J^{k} h_{m}-J^{k} g_{m} J^{k} h_{m}^{\sigma^{k}}=\left(J^{k} g_{m} \circ \sigma^{k}\right) J^{k} h_{m}-J^{k} g_{m}\left(J^{k} h_{m} \circ \sigma^{k}\right)=0 .
$$

Let $\tau \in \hat{G}$, its $k$-jet $\tau^{k}$ belongs to $G_{k}$. The group $H_{k}$ is Zariski-dense in $G_{k}$, therefore $\left(J^{k} g_{m} \circ \tau^{k}\right) J^{k} h_{m}-J^{k} g_{m}\left(J^{k} h_{m} \circ \tau^{k}\right)=0$. Then, we have

$$
J^{k}\left(g_{m}^{\tau} h_{m}-g_{m} h_{m}^{\tau}\right)=J^{k} g_{m}^{\tau^{k}} J^{k} h_{m}-J^{k} g_{m} J^{k} h_{m}^{\tau^{k}}=0,
$$

for every $k \in \mathbf{N}^{*}$, and passing to the projective limit on $k$, we obtain

$$
g_{m}^{\tau} h_{m}-g_{m} h_{m}^{\tau}=0
$$

and finally $f_{m}^{\tau}=f_{m}$.

LEMMA 10. - We assume that the VE are regular singular (it is always the case if we do not allow for equilibrium points or points at infinity). Let $m$ be a non-singular point of $\bar{\Gamma},(m \in \Gamma)$. If $f$ is a meromorphic first integral, then its germ $f_{m}$ at $\iota(m)$ is invariant by the differential Galois group $\hat{G}$.

Proof. - Let $\rho: \pi_{1}(\bar{\Gamma}, m) \rightarrow \operatorname{Diff}_{S p}^{\infty}(M, m)$ be the holonomy representation at $m$. We set $\hat{H}=\operatorname{Im} \rho$, the holonomy group at $m$. We have $\hat{H} \subset \hat{G}$. The first variational equation $V E=V E_{1}$ is regular singular. Therefore all the $V E_{k}$ are also regular singular. Then $H_{k}$ is Zariski-dense in $G_{k}$ (for every $k \in \mathbf{N}^{*}$ ) and $\hat{H}$ is Zariski-dense in $\hat{G}$. Then the result follows from the preceding lemma.

This lemma is the same as Proposition 13 (i), when $\Gamma=\bar{\Gamma}$ or when the singularities at the equilibrium points and at the points at infinity are regular-singular. If some of these singularities are irregular, we need to work a tad more.

The proof of the following lemma is a generalization of an argument used for a similar statement in the case of the $V E_{1}$ in [62].

LEMMA 11. - We allow for equilibrium points and points at infinity. If $f$ is a holomorphic first integral or a meromorphic first integral which is the quotient of two holomorphic functions defined on a neighbourhood of $\bar{\Gamma}$, then its germ $f_{m}$ at $m \in \Gamma$ is invariant by the differential Galois group $\hat{G}$.

Proof. - We begin with some notations and remarks. We choose a base point $m \in \Gamma$. We will work in an arbitrarily small simply connected neighbourhood $V$ of $m$ in $\Gamma$, and in an arbitrarily small neighbourhood $U$ of $\iota(m)$ in $M$, and we will assume that $\iota_{\mid V}$ is an embedding of $V$ in $U$. We "pull back" the germ $(M, \iota(m))$ as a germ $(M, m)$. We will choose the time parametrisation $t$ on $\Gamma$, with $t=0$ corresponding to $m$, and we will set $\iota(m)=\iota(0)$ for simplicity.

We return to the constructions of the beginning of this section. Let $k \in \mathbf{N}^{*}$ and $\bar{M}$ the completion of $M$ by a hypersurface at infinity. We recall that there exists a meromorphic trivialisation of the pullback on $\bar{\Gamma}$ of the meromorphic bundle of scalar $k$-jets on $\bar{M}$, compatible with the structure group $\operatorname{Diff}_{S p}^{k}(2 n ; \mathbf{C})$. We write this trivialisation $\overline{\bar{\Gamma}} \times J_{m}^{k}(M, m) \rightarrow \overline{\bar{\Gamma}}$. The differential system $L V E_{k}^{*}$ corresponds to $\nabla_{k}^{*}$ after the trivialisation. We denote by $a_{p}$ some coordinates on $J_{m}^{k}((M, m), \mathbf{C})$, identifying this jet algebra with $J_{0}^{k}\left(\mathbf{C}^{2 n}, \mathbf{C}\right)$ ( $p$ is a multiindex). We denote by $\phi_{t}:(M, m) \rightarrow(M, \iota(t))$ the flow on $U$, with $\phi_{0}=\operatorname{id}_{(M, m)}$, and we identify it with the corresponding map $\phi_{t}:(M, 0) \rightarrow(M, t)$. We set $\psi_{t}=\phi_{t}^{-1}$ as before. The entries of the matrix versions of $J^{k} \phi_{t}=\phi_{t}^{k}$ and $J^{k} \psi_{t}^{k}=\psi_{t}^{k}$ corresponding to our coordinate choice are holomorphic on $V$ and they belong to the Picard-Vessiot extension $L_{k}$ of $k_{\bar{\Gamma}}$. 
Let $f^{(k)} \in J_{m}^{k}((M, m), \mathbf{C})$ be a scalar $k$-jet. We set $f^{(k), t}=f^{(k)} \circ \psi_{t}^{k}$. We have $f^{(k), t} \in$ $J_{m}^{k}\left((M, \iota(t))\right.$ and we identify it with an element of $J_{m}^{k}((M, t))$. Then $t \rightarrow f^{(k), t}$ is a solution of the $L V E_{k}^{*}$ on $V$ and the corresponding holomorphic functions $a_{p}(t)$ belong to the PicardVessiot extension $L_{k}$ of $k_{\bar{\Gamma}}$. Let $G\left(L V E_{k}\right)$ be the differential Galois group of $L V E_{k}^{*}$ associated to this Picard-Vessiot extension, $\sigma^{k} \in G\left(L V E_{k}\right)$ acts on each of the $a_{p}(t)$, and we obtain a holomorphic family $g^{(k), t}$ of $k$-jets on $V$, whose coordinates are the $\sigma^{k}\left(a_{p}\right)(t)$. If we set $g^{k}=g^{(k), 0}$, then $g^{(k), t}=g^{(k)} \circ \psi_{t}^{k}\left(t \mapsto g^{(k), t}\right.$ is also a solution of the $L V E_{k}^{*}$ on $\left.V\right)$. We consider $\left(\psi_{t}^{k}\right)^{\sigma^{k}} \circ \psi_{t}^{k^{-1}}=\left(\psi_{t}^{k}\right)^{\sigma^{k}} \circ \phi_{t}^{k}$. It is independent of $t$ and it is equal to the natural image $\rho_{k}\left(\sigma^{k}\right)$ of $\sigma^{k}$ in $\operatorname{Diff}_{S p}^{k}(M, 0)$ (cf. (29)). If we identify $\rho_{k}\left(\sigma^{k}\right)$ and $\sigma^{k}$, we obtain finally $g^{(k)}=f^{(k)} \circ \sigma^{k}=\left(J^{k} f\right)^{\sigma^{k}}$.

We will return now to the proof of Lemma 11.

First case. We will start by assuming that the first integral $f$ is holomorphic.

To a holomorphic function $f$ on $U$, we can associate the holomorphic family of germs of holomorphic functions $t \mapsto f^{t}=f_{0} \circ \psi_{t}$, on $V$. The germ $f^{t}$ is a germ at $\iota(t) \in M$, and we can identify it with a germ "at $t$ " (i.e. on $(M, t)$ ). We have $f^{0}=f_{m}=f_{0}$. The function $f$ is a first integral of $X=X_{H}$ if and only if $f^{t}=f_{\iota(t)}, f_{\iota(t)}$ being the germ at $\iota(t) \in U$ of the holomorphic function $f$.

Let $k \in \mathbf{N}^{*}$. We set $J^{k} f=f^{(k)}$ and we do the preceding constructions. If $\sigma^{k} \in G\left(L V E_{k}^{*}\right)=$ $G_{k}$, we have $g^{(k), t}=f^{(k)} \circ\left(\psi_{t}^{k}\right)^{\sigma^{k}}=\left(J^{k} f^{t}\right)^{\sigma^{k}}$ and $g^{(k)}=J_{0}^{k} f \circ \sigma^{k}$. If moreover $f$ is the restriction to $U$ of a first integral which is holomorphic on a neighbourhood of $\iota(\bar{\Gamma})$ in $\bar{M}$, then $g^{(k), t}=\left(J^{k} f^{t}\right)^{\sigma^{k}}=\left(J^{k} f_{\iota(t)}\right)^{\sigma^{k}}$ on $V$, but $x \rightarrow J_{\iota(x)}^{k}(x \in \bar{\Gamma})$ is holomorphic on $\Gamma$ and it extends to a meromorphic function on $\bar{\Gamma}$. Therefore, the coordinates $a_{p}$ of $J^{k} f^{t}=J^{k} f_{\iota(t)}$ extend to meromorphic functions on $\bar{\Gamma}$. That is, they are elements of the field $k_{\bar{\Gamma}}$. Then $\left(J^{k} f_{\iota(t)}\right)^{\sigma^{k}}=$ $J^{k} f_{\iota(t)}=J^{k} f^{t}, g^{(k), t}=J^{k} f^{t}, g^{(k)}=J_{0}^{k} f$ and finally

$$
J_{0}^{k} f=J_{0}^{k} f \circ \sigma^{k} .
$$

Therefore, for every $k \in \mathbf{N}^{*}$, the $k$-jet at $m$ of a first integral $f$, which is holomorphic on a neighbourhood of $\iota(\bar{\Gamma})$ in $M$, is invariant by the group $G_{k}$. Taking projective limit in $k, f$ (i.e. its infinite jet) is therefore invariant by $\hat{G}$.

Second case. We will assume now that the first integral $f$ is meromorphic and it is the quotient of two functions $g$ and $h$ which are defined and holomorphic on a neighbourhood of $\bar{\Gamma}$. We set, as before, $f^{t}=f_{0} \circ \psi_{t}, g^{t}=g_{0} \circ \psi_{t}, h^{t}=h_{0} \circ \psi_{t}$. If $f$ is a first integral, then $f^{t}=f_{\iota(t)}$, therefore $\frac{g^{t}}{h^{t}}=\frac{g_{\iota(t)}}{h_{\iota(t)}}$ and $g^{t} h_{\iota(t)}-g_{\iota(t)} h^{t}=0$.

Let $k \in \mathbf{N}^{*}$. Then we have: $J^{k} g^{t} J^{k} h_{\iota(t)}-J^{k} g_{\iota(t)} J^{k} h^{t}=0$, in the $k$-jet sense. The coordinates of the jets $J^{k} g_{\iota(t)}$ and $J^{k} h_{\iota(t)}$, are holomorphic functions on $V$ and they extend to meromorphic functions on $\bar{\Gamma}$, that is, they belong to the field $k_{\bar{\Gamma}}$. The coordinates of the jets $J^{k} g^{t}$ and $J^{k} f^{t}$ belong to the Picard-Vessiot extension $L_{k}$ of $k_{\bar{\Gamma}}$.

Let $\sigma^{k} \in G\left(L V E_{k}^{*}\right)=G^{k}$. We apply it to $J^{k} g^{t} J^{k} h_{\iota(t)}-J^{k} g_{\iota(t)} J^{k} h^{t}=0$ and we get $\left(J^{k} g^{t}\right)^{\sigma^{k}} J^{k} h_{\iota(t)}-J^{k} g_{\iota(t)}\left(J^{k} h^{t}\right)^{\sigma^{k}}=0$. Then (copying what we did in the first case and taking $t=0)$ we obtain

$$
J^{k}((g \circ \sigma) h-g(h \circ \sigma))=\left(J^{k} g \circ \sigma^{k}\right) J^{k} h-J^{k} g\left(J^{k} h \circ \sigma^{k}\right)=0 .
$$

Let $\sigma \in \hat{G}$. We set $\sigma^{k}=J^{k} \sigma \in G_{k}$ and taking the projective limit on $k$ of the relations (31), we have $(g \circ \sigma) h-g(h \circ \sigma)=0$ and $f=\frac{g}{h}=\frac{g \circ \sigma}{h \circ \sigma}=f \circ \sigma=f^{\sigma}$. Then $f$ is invariant by $\hat{G}$.

We now consider a local case.

$4^{\text {e }}$ SÉRIE - TOME $40-2007-\mathrm{N}^{\circ} 6$ 
LEMMA 12. - Let $f$ be a meromorphic first integral on a neighbourhood $U^{\prime} \subset \bar{M}$ of an equilibrium point or of a point at infinity $\iota(a) \in \bar{M}(a \in \bar{\Gamma} \backslash \Gamma)$. Let $V^{\prime}$ be a simply connected neighbourhood of a in $\hat{\Gamma}$, such that $\iota\left(V^{\prime}\right) \subset U^{\prime}$. Let $m \neq a$ be a point of $V^{\prime}$. If $f$ is a meromorphic first integral, then its germ $f_{m}$ at $m$ is invariant by the local differential Galois group $\hat{G}(a)$.

Proof. - In this statement, we interpret "the" local differential Galois group at $a$, which depends on the choice of a "base point"- for instance a direction issued from $a$ on the tangent space $T_{a}(M)$-as a subgroup of $\operatorname{Diff}_{S p}^{\infty}(M, m)$. If $m^{\prime} \neq a$ is another point of $V^{\prime}$, then the analytic continuation along an arbitrary continuous path from $\iota(m)$ to $\iota\left(m^{\prime}\right)$, on $\iota\left(V^{\prime}\right)$, induces an isomorphism between $\operatorname{Diff}_{S p}^{\infty}(M, m)$ and $\operatorname{Diff}_{S p}^{\infty}\left(M, m^{\prime}\right)$, depending of course on the homotopy class of the path in $V \backslash\{a\}$ ). Then, for the proof of the lemma, we can change $m$ arbitrarily and replace $U^{\prime}$ by a smaller neighbourhood. Therefore we can assume that $f$ is the quotient of two functions $g$ and $h$ which are holomorphic on $U$ and that $V^{\prime} \backslash\{a\}$ is a connected component of $\iota^{-1}\left(U^{\prime} \backslash\{\iota(a)\}\right.$, and we can subsequently apply Lemma 11 (replacing $\bar{\Gamma}$ by $V^{\prime}$ and $\bar{M}$ by $U^{\prime}$ ).

We can now prove the statement (i) of the Proposition 13 in the (most general) meromorphic case. We assume $f$ is a meromorphic first integral on a neighbourhood of $\iota(\bar{\Gamma})$ in $\hat{M}$. Let $m$ be a point of $\Gamma$. We consider the differential Galois group $\hat{G} \subset \operatorname{Diff}_{S p}^{\infty}(M, m)$. We can identify the Galois group $\hat{G}^{\prime}$ of the restriction to $\Gamma$ of our "system" with a subgroup of $\hat{G}$ and "interpret" the local Galois groups $\hat{G}(a)(a \in \hat{\Gamma} \backslash \Gamma)$ as subgroups of $\hat{G}$, using a finite set of continuous paths (on $\Gamma$ ) from $m$ to some points which are "sufficiently near" to the points $a$-of course, this is generally not canonical, but such fact is of no importance here. Then the germ $f_{m}$ is invariant by $\hat{G}^{\prime}$ (cf. Lemma 11) and by the local groups $\hat{G}(a)$, up to our interpretation (cf. Lemma 12). For every $k \in \mathbf{N}^{*}$, the differential Galois group $G_{k}$ is the Zariski closure of the subgroup generated by the elements of $G_{k}^{\prime}$ and $G(a)_{k}$ (cf. [62]), therefore $\hat{G}$ is the Zariski closure of the subgroup generated by $\hat{G}^{\prime}$ and the $\hat{G}(a)$. Using Lemma 9, we obtain the invariance of $f_{m}$ by $\hat{G}$. This ends the proof of Proposition 13.

Now we can finish the proof of our main theorem. With the hypotheses of this theorem, the Lie algebra $\hat{\mathcal{G}}$ is symplectic and orthogonal to the germs $f_{1}, \ldots, f_{n}$. Therefore it is abelian (see Theorem 3) and by Proposition 12 (ii) the Lie algebra $\mathcal{G}^{k}$ must be also abelian.

\section{Obstructions to integrability and non-linear Galois theory}

Whenever one wants to prove the non-integrability of some Hamiltonian system, having to choose a "nice" solution of the system (a solution we can parametrise using some "special functions") sounds a priori quite strange. It seems on the contrary reasonable to think of solutions of non-integrable systems as "very transcendental". In fact in all the applications done of our theory up to now, this is not the case: the "generic solution" of the system is presumably highly transcendental (there are few precise results in this direction), but there are invariant integrable subsystems giving interesting "nice" solutions. Perhaps this is due to the fact that applications are done to simple enough systems.

However, from a theoretical point of view, it remains interesting to try to avoid the choice of a particular solution $\Gamma$. In order to do this, a natural tool is the non-linear differential Galois theory. This theory was introduced by J. Drach $[25,26]$, and developed later by J. Drach and E. Vessiot. J. Drach studied some applications of his theory to various questions of geometry and mechanics such as the spinning top. P. Painlevé gave a "proof" of the fact that Painlevé transcendents are "new transcendents" using Drach's theory. Unfortunately there are important gaps in the foundations of Drach's theory, and in its applications. Therefore, when we began to work on the present paper some years ago, no satisfactory non-linear Galois theory was available. 
The situation is completely different nowadays, as we have two such theories due respectively to H. Umemura [79] and B. Malgrange [56,57]. We will explain the analogue of our main theorem using Malgrange's approach (the similar result must be true with Umemura's approach, but this is conjectural). This analogue is due to the second author (unpublished). We will be very sketchy; the interested reader will find some details about this result, and the necessary definitions and theorems in $[56,57,20]$ (cf. in particular [20, 5.4]).

The main tool due to $\mathrm{B}$. Malgrange is the notion of Lie $\mathcal{D}$-groupoid on a complex analytic manifold $M$. Roughly speaking it is a sub-groupoid of the groupoid $\mathcal{M}$ of germs of analytic diffeomorphisms of $M$ defined by analytic PDE. We recall that a groupoid is a (small) category whose all the morphisms are isomorphisms. Here we start with the groupoid $\mathcal{M}$ whose objects are the points $(a, b, \ldots)$ of $M$ and the morphisms are the invertible germs $g$ of analytic maps $g:(M, a) \rightarrow(M, b)(f(a)=b)$. A $\mathcal{D}$-groupoid has a $\mathcal{D}$-Lie algebra defined by PDE. B. Malgrange defines the Galois $\mathcal{D}$-groupoid or $\mathcal{D}$-hull of an analytic dynamical system (differential equation, foliation, etc.) as the smallest $\mathcal{D}$-groupoid such that "its Lie algebra contains the infinitesimal transformations of the dynamics" (more precisely these transformations must be solutions of the $\mathcal{D}$-Lie algebra). The (very) difficult point is whether such a smallest groupoid exists. This definition is, a posteriori, very natural: "that which algebra sees from the dynamics", as B. Malgrange puts it. It is related to the idea that there is no "Lie third theorem" for Lie $\mathcal{D}$-groupoids.

For an autonomous system we get the smallest $\mathcal{D}$-groupoid such that its "Lie algebra contains the corresponding vector field".

The computation of the Galois $\mathcal{D}$-groupoid seems extremely difficult (even for apparently "simple" cases as Painlevé equations). There are only partial theoretical results (cf. in particular [20]) and nothing is effective as there are no algorithms. Therefore the following result is, for the moment, quite agreeable, albeit theoretical.

As for the computation of differential Galois groups in the linear case, a natural idea is to try to reduce the problem using "majorants" of the Galois $\mathcal{D}$-groupoid, i.e. $\mathcal{D}$-groupoids containing the Galois $\mathcal{D}$-groupoid.

THEOREM 6. - Let $(M, \omega)$ be a connected symplectic analytic manifold of dimension $2 n$. Let $H: M \rightarrow \mathbf{C}$ be an analytic function, the Hamiltonian. We assume that the corresponding Hamiltonian system is completely meromorphically integrable. Then the $\mathcal{D}$-Lie algebra of the Galois $\mathcal{D}$-groupoid of the system (i.e., the $\mathcal{D}$-hull of the vector field $X_{H}$ ) is abelian.

An evident majorant of our Galois $\mathcal{D}$-groupoid is the $\mathcal{D}$-groupoid defined by the analytic system of PDE:

$$
g^{*} f_{i}=f_{i}, \quad i=1, \ldots, n, \quad g^{*} \omega=\omega .
$$

Its linearisation is

$$
L_{X} f_{i}=0, \quad i=1, \ldots, n, \quad L_{X} \omega=0 .
$$

This $\mathcal{D}$-Lie algebra is clearly abelian. This follows from Theorem 3. (We need only the simple meromorphic version, not the formal version.) Then Theorem 6 follows immediately.

One works with $\mathcal{D}$-groupoids systematically on the "complementary of a hypersurface" (in some "algebraic" delicate sense). On the contrary our theory (more precisely its applications) is (are) centred on the choice of non-generic solutions $\Gamma$, which in general will live into the exceptional hypersurfaces. Therefore a comparison between both approaches seems difficult. We will only suggest an analogy: in terms of topology of foliations, Galois $\mathcal{D}$-groupoids will correspond to holonomy groupoids, our $\Gamma$ (the "interesting" one) will correspond to holonomy carriers (exceptional leaves).

$4^{\text {e }}$ SÉRIE - TOME $40-2007-\mathrm{N}^{\circ} 6$ 


\section{On the applications}

From now on we identify the linearised variational equation with the variational equation $V E_{k}$ in a further abuse of notation. We will give some indications and references about a few interesting applications of our main result (Theorem 5).

A typical situation [59] is the following:

- the Riemann surface $\Gamma$ is a punctured elliptic curve, the corresponding elliptic curve being denoted as $\bar{\Gamma}=\Gamma \cup\{\infty\}$;

- the extension to $\bar{\Gamma}$ of the first variational equation is regular singular.

In the classical applications parametrised families of Hamiltonian systems appear. With the above hypotheses we get parametrised families of regular singular equations on $\bar{\Gamma}$ (the corresponding $V E=V E_{1}$ ), and in many cases we obtain obstructions to integrability for all the values of the parameter except for a finite or discrete subset using theorem [62] (that is the first $V E$ ). The exceptional values of the parameter correspond typically to direct sums of LaméHermite equations where each of the equations fall in the Lamé case with one solution in the field of elliptic functions of the coefficients (see [59], page 147). In such a situation the identity component of the differential Galois group $G_{1}$ of $V E_{1}$ is commutative (isomorphic to an additive group $\mathbf{C}^{p}$ ) and there is no obstruction.

We denote by $\wp$ the Weierstrass function corresponding to a double pole at the origin of $\mathbf{C}$ $(\wp(0)=\infty \in \bar{\Gamma})$. Then a Lamé equation is a linear ODE

$$
\frac{d^{2} \xi}{d t^{2}}=(A \wp(t)+B) \xi
$$

The unique singular point is the origin modulo periods of the Weierstrass $\wp$ function, that is $\infty \in \bar{\Gamma}$. The equation is regular singular. The classical notation is $A=n(n+1)$.

The differential Galois group of (34) is commutative if and only if $n \in \mathbf{Z}$. It is the LaméHermite case. In that case there exists a meromorphic solution (an elliptic one, more precisely) and the differential Galois group is triangular. Its elements are represented by unipotent triangular $2 \times 2$ matrices of the form

$$
\left(\begin{array}{ll}
1 & 0 \\
\alpha & 1
\end{array}\right)
$$

It is trivial or isomorphic to the additive group $\mathbf{C}$. Therefore it is commutative and connected.

We assume now that the $V E$ is a direct sum of Lamé-Hermite equations. For the sake of simplicity we can consider the case of two such equations:

$$
\left(\begin{array}{lllll}
1 & 0 & 0 & 0 & \\
\alpha & 1 & 0 & 0 & \\
0 & 0 & 1 & 0 & \\
0 & 0 & \beta & 1 & \\
& & & & \ddots
\end{array}\right) .
$$

The $2 \times 2$ unipotent matrices in the elements of $G_{1}$ give us the Galois group of each of the Lamé equations (34). It is either trivial or the additive group C. As a consequence $G_{1}$ is a commutative connected group.

The variational equation $V E_{1}$ is regular singular. Therefore all the higher-order variational equations $V E_{k}$ (more precisely their linear counterparts $L V E_{k}$ ) are also regular singular (for 
$k \geqslant 1$ ) and their Galois groups $G_{k}$ are given by the Zariski closure of their monodromy groups. Furthermore, $G_{k}$ is connected because $G_{1}$ is connected. We have the following lemma.

LEMMA 13. - Assume that the first-order variational equation $V E_{1}$ decomposes in a direct sum of Lamé-Hermite type equations. Then $G_{k}$ is commutative if and only if the solutions of $V E_{k}$ are meromorphic functions on the covering $\mathbf{C}$ of $\bar{\Gamma}$.

The proof is simple. The monodromy group of each of the $V E_{k}$ is a linear representation of the fundamental group of $\Gamma=\bar{\Gamma} \backslash\{\infty\}$ (the point $\infty$ is represented in the Weierstrass parametrisation by the origin modulo periods) and this fundamental group is free, non-commutative and generated by the translations along the periods. The commutator of these two generators is represented by a simple loop around the singular point $\infty$. Hence, a monodromy group is commutative if and only if the monodromy associated to this simple loop is trivial. By Zariski closure, a differential Galois group $G_{k}$ is commutative if and only if the corresponding monodromy subgroup is commutative. Therefore we can check the commutativity of $G_{k}$ locally at $\infty$. Recursively, by local power series expansions of the solutions of $V E_{k-1}$ and quadratures, it is easy to check if $V E_{k}$ has solutions which ramify around 0 . One only needs to check for the existence of a residue different from zero, which will give rise, by integration, to a local logarithm.

In [49], the authors completely solved the problem of meromorphic integrability of twodegrees of freedom Hamiltonian systems with homogeneous potentials of degree three. After reduction, the problem boils down to the study of the 2-parameter family of potentials

$$
V\left(x_{1}, x_{2}\right)=\frac{1}{3} a x_{1}^{3}+\frac{1}{2} x_{1}^{2} x_{2}+\frac{1}{3} c x_{2}^{3}, \quad a, b \in \mathbf{C} .
$$

The authors made a considerable use of Theorem 1-see their Introduction-exploiting the commutativity of $\left(G_{1}\right)^{0}$. More precisely, they used a corollary of it stated in [64]. Moreover, several of the subfamilies for which no obstruction to integrability is obtained from the firstorder analysis are well-known integrable systems, except the 1-parametric subfamily with potential $c=1$ and arbitrary $a$ in (37). In order to study the meromorphic integrability of this subfamily, the authors applied the main theorem of the present paper, Theorem 5, and checked the commutativity of the identity component of the Galois groups of the higher-order variational equations: if one of them is non commutative, the system is not integrable with meromorphic first integrals. It is interesting to point out that the problem of the integrability of a member of this subfamily (i.e., for $a=0$ ) was considered as an open problem in the recent monograph [2] (page 180).

They used the above mentioned Lamé-Hermite approach. Back to the Hamiltonian systems with cubic potential (37), the first-order variational equations decompose in two Lamé equations with Galois group $G_{1}$ given by $4 \times 4$ matrices such as (36). In [49] a residue different from zero is obtained for the integrand of a solution of the second-order variational equation if $a \neq 0$ (resp. of the third-order variational equation if $a=0$ ). Then $G_{2}=\left(G_{2}\right)^{0}$ (resp. $G_{3}=\left(G_{3}\right)^{0}$ ) is non-commutative and this family is non-integrable.

Along the same lines, it is possible to prove that the two-degree-of-freedom Hamiltonian system defined by the cubic Hamiltonian

$$
H=\frac{1}{2}\left(y_{1}^{2}+y_{2}^{2}\right)+\frac{1}{2} x_{1}^{2}+\frac{1}{2} x_{2}^{2}+\frac{1}{3} x_{1}^{3}+\frac{1}{2} x_{1} x_{2}^{2}
$$

is non-integrable. This system belongs to the Hénon-Heiles family of one-parameter Hamiltonians considered by Ito [35]. By means of the first-order variational equation it was proved that

$4^{\mathrm{e}}$ SÉRIE - TOME $40-2007-\mathrm{N}^{\circ} 6$ 
for all except four of the values of the parameter, the systems in this family are non-integrable, see $[35,65,59]$. Three of these remaining cases are trivially integrable. The fourth case is (38) and its non-integrability was conjectured from numerical experiences. But every single attempt at a rigorous proof of this fact has been unsuccessful during the last years. Now we can prove the non-integrability of this last case, using our main theorem and the Lamé-Hermite approach. We get an obstruction (a non-trivial residue) for the third variational equation. See the details in Appendix B.

In this way it is possible to close the problem of integrability for the Hénon-Heiles family of Ito. This example was in fact the motivation for the Section 8.3.2 in [59] and it also was the initial motivation for the present paper, following an idea of the third author.

\section{Open problems}

The main theorem in this paper is a necessary condition for integrability of Hamiltonian systems by meromorphic first integrals: the identity components of all the Galois groups $G_{m}$ of the higher-order variational equations, $m \geqslant 1$, must be commutative. This gives an infinite number of conditions to be satisfied. It is now very natural to ask whether or not these necessary conditions for meromorphic integrability, if fulfilled simultaneously, are also sufficient. This problem was already formulated in [59], p. 146, in the following manner. Assume that the identity components of the Galois groups $G_{k}$ of all the variational equations $V E_{k}$ of order $k \geqslant 1$ (or, more precisely, their linear counterpart $L V E_{k}$ ) are commutative, then:

Is the Hamiltonian system $X_{H}$ completely integrable with meromorphic first integrals in some neighbourhood of the completed integral curve represented by the Riemann surface $\bar{\Gamma}$ ?

We remark that, without any further additional assumption about $X_{H}$ (or about $\Gamma$ ), the answer to this problem is negative.

An example of a non-integrable system with an integral curve such that all the groups $G_{k}$ are commutative, is the planar three-body problem along the parabolic solutions of Lagrange, see $[14,78]$. When the angular momentum of the bodies is zero, the Galois groups of all the variational equations $V E_{k}$ are commutative. The fundamental group of the Riemann surface $\Gamma$ defined by such a solution is commutative: $\Gamma$ is the Riemann sphere with two points deleted. The variational equations $V E_{k}$ are of Fuchs class and their monodromy groups, which are representations of this fundamental group, are also commutative. Since the Galois groups $G_{k}$ are given by the Zariski adherences of the corresponding monodromy groups, they are also commutative.

So, the problem is to understand if it is possible to impose some natural generic conditions in order to get a positive answer to our question:

Problem. - Let $X_{H}$ be a complex analytical Hamiltonian system defined over a complex analytical symplectic manifold and let $\Gamma$ be the immersed Riemann surface defined by a particular integral curve of $X_{H}$ which is not reduced to an equilibrium point.

Under which conditions is $X_{H}$ completely integrable with meromorphic first integrals in some neighbourhood of the completed integral curve represented by the Riemann surface $\bar{\Gamma}$, provided the identity components of the Galois groups $G_{k}$ are commutative for all $k$ ?

Another problem concerns the dynamical implications of non-integrability. In two-degree-offreedom systems a typical effect of non-integrability is the existence of transversal homoclinic 
orbits. It is well known that this kind of orbits prevents from the existence of analytic first integrals in a vicinity of the orbit. See [67] for a general exposition. For early applications to the three-body problem see [41-43]. In [65] the non-integrability of some systems is illustrated by the existence of hyperbolic periodic orbits whose invariant manifolds have transversal intersection. In this example there is a strong numerical evidence that such orbits do not exist in real phase space. For this reason the search for homoclinic orbits was done in the complex phase space. In systems with a larger number of degrees of freedom the problem is even more subtle.

It seems natural to ask for the following question:

Problem. - Assume a complex analytical Hamiltonian is proved to be non-integrable by the methods presented in this paper.

Is it true that some transversal homoclinic orbit to an invariant object exists?

Finally, there is another kind of related problems worth clarifying. In the seminal work [33] not only numerical evidence of non-integrability was given, but also some quantitative information on the lack of integrability. This has primordial relevance for physical applications. The method used was the computation of an indicator, analogous to the maximal Lyapunov exponent, such that it takes the value zero in ordered orbits and is positive on chaotic orbits. This allows to define a fraction of integrability on selected levels of the energy. For instance, for the HénonHeiles system the system can be considered integrable for any practical purpose for energies in the range [0,0.05].

For small perturbations of an integrable system (e.g., near a totally elliptic fixed point in a general system, where the integrable approximation is a Birkhoff normal form) it is well known that the lack of integrability is exponentially small in the small parameter. See [70] for upper bounds using averaging theory and [30,31] for upper bounds of the related splitting. Equivalently to a quantitative measure of the lack of integrability, we can look for the existence of quasiintegrals, which are approximately preserved in the real phase space.

On the other hand, most of the proofs of non-integrability make use of special orbits with singularities for some $t \in \mathbf{C}$. The measures of splitting use also, typically, the behaviour of invariant manifolds in a neighbourhood of these singularities. Then, the next question, which is certainly related to [82], seems relevant:

Problem. - Assume some singularities are used to detect non-integrability by applying the main theorem of this paper.

What kind of information is also needed to produce quantitative estimates of the lack of integrability? (e.g., to give estimates of a suitable splitting, or of the measure of the domain with positive maximal Lyapunov exponent, or of the metric entropy, in the real phase space).

\section{Acknowledgements}

The authors are indebted to Nguyen Tien Zung for pointing out a technical question concerning the proof of Theorem 5 in a preliminary version of the paper and to Sergi Simon for language help. The second author thanks Bernard Malgrange for useful remarks. The work of first author has been supported by grant DGICYT BFM2003-09504-C02-02 (Spain). The second author has been partially supported by the NEST EU Math. Project GIFT, FP6-005006-2. The work of third author has been supported by grants DGICYT BFM2003-09504-C02-01, MTM200605849/Consolider (Spain) and CIRIT 2005 SGR-1028 (Catalonia).

$4^{\text {e }}$ SÉRIE - TOME $40-2007-\mathrm{N}^{\circ} 6$ 


\section{Appendix A. A trivialisation theorem}

In order to apply differential Galois theory to the higher variational equations $L V E_{k}$, we need to replace these connections by ordinary differential systems, that is to trivialise (meromorphically) our jet bundles. We can avoid this problem if we use the Tannakian approach of the differential Galois theory [23].

Let $k, n \in \mathbf{N}$. Let $E=\mathbf{C}^{2 n}$. We set $\operatorname{Diff}_{S p}^{k}(2 n ; \mathbf{C})=\operatorname{Diff}_{S p}^{k}(E)$. For $k=1$, we have $\operatorname{Diff}_{S p}^{1}(2 n ; \mathbf{C})=S p(2 n ; \mathbf{C})$. We recall that $\operatorname{Diff}_{S p}^{1}(2 n ; \mathbf{C})$ is the semi-direct product of $S p(2 n ; \mathbf{C})$ and a unipotent linear algebraic group $U^{k}(2 n ; \mathbf{C})=U^{k}(E)$. Let $X$ be a connected Riemann surface. Let $G$ be a complex linear algebraic group. In [62], Appendix A, we defined locally trivial meromorphic bundles over $X$ admitting $G$ as structure group.

Proposition 14. - Let $X$ be a complex connected, non-compact Riemann surface and let $(\mathcal{F}, p, X)$ be a locally trivial holomorphic vector bundle over $X$ having $\operatorname{Diff}_{S p}^{k}(2 n ; \mathbf{C})$ as structure group. Then, $\mathcal{F}$ is holomorphically trivial.

The algebraic group $\operatorname{Diff}_{S p}^{k}(2 n ; \mathbf{C})$ is connected for the ordinary topology $(S p(2 n ; \mathbf{C})$ and $U^{k}(2 n ; \mathbf{C})$ are connected for this topology). Then the proposition follows from a theorem by Grauert ([62], Appendix A, Theorem A.1).

PROPOSITION 15. - Let $X$ be a complex connected compact Riemann surface. Let $(\mathcal{F}, p, X)$ be a locally trivial holomorphic vector bundle over $X$ with structure group $\operatorname{Diff}_{S p}^{k}(2 n ; \mathbf{C})$. Then $\mathcal{F}$ is meromorphically trivial.

We will resort to comparison between analytic bundles and algebraic bundles (in "GAGA" style). It is equivalent to work with principal bundles. For a complex algebraic manifold $X$ (resp. a complex linear algebraic group $G$ ) we denote $X^{h}$ the corresponding analytic manifold (resp. $G^{h}$ the corresponding analytic group). Then every principal bundle with basis $X^{h}$ and group $G^{h}$ is algebraic (cf. [74, Théorème 3, pp. 1-34]). In general this algebraic bundle is not locally trivial (in the algebraic sense); it is only locally isotrivial (that is, trivial up to a finite unbranched covering). However this bundle is trivial if the algebraic group $G$ is special (cf. below). Then, Proposition 15 will follow from the fact that the algebraic group Diff ${ }_{S p}^{k}(2 n ; \mathbf{C})$ is special (cf. Corollary 4 below).

DEFINITION 3. - Let $G$ be a complex algebraic group. We will say that it is special if every principal bundle with group $G$ is locally trivial.

Every special group is connected and linear (cf. [73, Théorème 1]). We have the following result (cf. [73, Théorème 2, pages 1-24]).

THEOREM 7. - An algebraic subgroup $G$ of $G l(n ; \mathbf{C})$ is special if and only if the following condition is satisfied:

(R) there exists a regular section of the fibration $G l(n ; \mathbf{C}) / G \rightarrow G l(n ; \mathbf{C})$.

THEOREM 8.-

(i) The group $S p(2 n ; \mathbf{C})$ is special.

(ii) Every connected solvable linear algebraic group is special.

(iii) Let $G$ be an algebraic group and $H$ be an invariant subgroup. If $H$ and $G / H$ are special algebraic groups, then $G$ is special.

(i) If $G=S p(2 n ; \mathbf{C})$, the homogeneous space is the space of antisymmetric non-degenerate 2-forms $\sum_{i<j} a_{i j} x_{i} \wedge x_{j}$, and the condition ( $\mathrm{R}$ ) is satisfied: the generic form 
$\sum_{i<j} u_{i j} x_{i} \wedge x_{j}$ is equivalent to the canonical form $\sum_{i=1}^{n} x_{2 i-1} x_{2 i}$ up to a linear coordinate change with coefficients in the field $\mathbf{C}\left(u_{i j}\right)$ (cf. [73, page 34]).

(ii) It is a result of Rosenlicht (cf. [73]), [74].

(iii) Cf. [74, Lemme 6, pages 1-25].

COROLlaRY 4. - The algebraic group $\operatorname{Diff}_{S p}^{k}(2 n ; \mathbf{C})$ is special.

This completes the proof of Proposition 15.

\section{Appendix B. Proof of non-integrability of system (38)}

This special case of the Hénon-Heiles problem has only two fixed points: one of them, totally elliptic, located at the origin; the other fixed point, $P_{h p}$, located at $x_{1}=-1, x_{2}=y_{1}=y_{2}=0$ on the level $H=h^{*}=1 / 6$, is of hyperbolic-parabolic type. We note that the plane $x_{2}=y_{2}=0$ is invariant. On that plane and on the level $H=h^{*}$ the system has a separatrix, tending to $P_{h p}$ for $t \rightarrow \pm \infty$ on the real phase space. We shall make use of this special solution. The fact that the point $P_{h p}$ has a degeneracy is certainly related to the difficulties in proving non-integrability for this system.

Working on the real phase space, a Poincaré section through $x_{2}=0$ on the bounded component of the level $H=h$, for $h \in\left(0, h^{*}\right)$, displays only a tiny amount of chaoticity. However, for energies $h>h^{*}$ the chaotic domain is clearly visible. Fig. 1 shows an illustration for $h=1 / 5$. The point marked as $Q$ corresponds to an hyperbolic periodic orbit. It belongs to a family born at $h=h^{*}$.

The special solution $\Gamma$ is given by

$$
x_{1}(t)=\frac{3 / 2}{\cosh ^{2}(t / 2)}-1, \quad y_{1}(t)=\frac{-(3 / 2) \sinh (t / 2)}{\cosh ^{3}(t / 2)}, \quad x_{2}=y_{2}=0,
$$

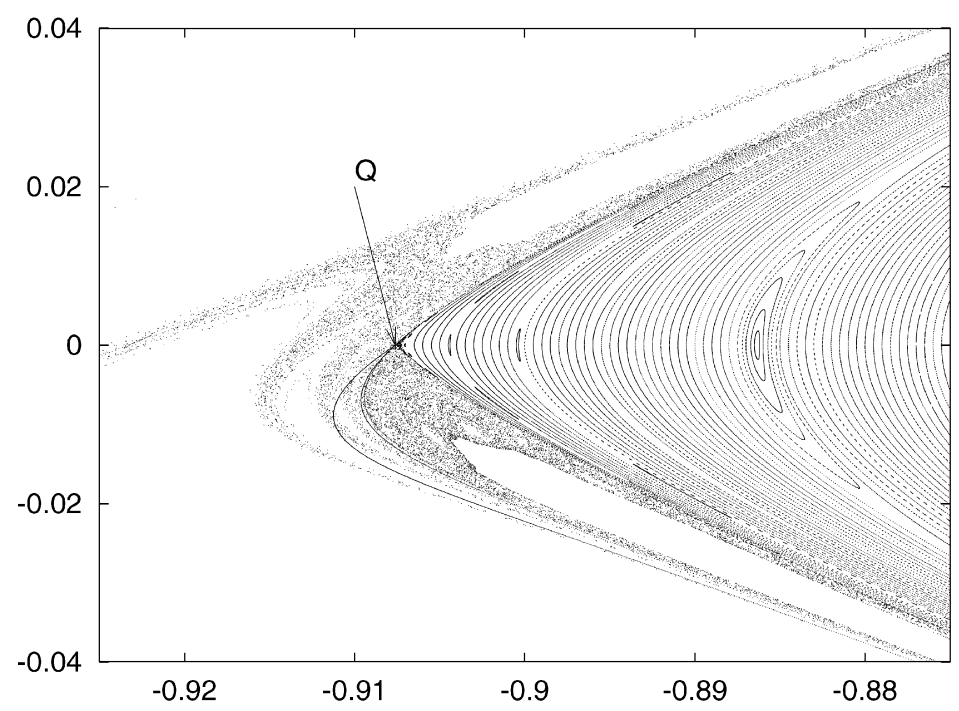

Fig. 1. Part of a Poincaré section of (38) through $x_{2}=0$ in the energy level $h=1 / 5$, displayed in the $\left(x_{1}, y_{1}\right)$ variables. Initial points are taken on $y_{1}=0$ and 20,000 iterates are computed for each initial point, keeping only the ones in the window shown.

$4^{e}$ SÉRIE - TOME $40-2007-\mathrm{N}^{\circ} 6$ 
where the origin of time has been taken on $y_{1}=0$, to have a symmetric expression. It has singularities for $\cosh (t / 2)=0$, i.e., for $t=(2 k+1) \pi \mathbf{i}$, with $k \in \mathbf{Z}$. Our approach starts by computing the third-order monodromy, that is, the solution of the variational equations up to order three, starting at the point $\left(x_{1}(0), y_{1}(0)\right)$, along a path $\gamma \subset \Gamma$ which encloses only the singularity $t=\pi \mathrm{i}$ and has index 1 with respect to it. It is clear that the result is independent on the path. For concreteness we introduce the following notation (see (12))

(i) $x_{3}=y_{1}, x_{4}=y_{2}$,

(ii) the components $D_{k} x_{j}$ will be denoted as $x_{j, k}$. In a similar way, the components $D_{k_{1}, k_{2}}^{2} x_{j}, D_{k_{1}, k_{2}, k_{3}}^{3} x_{j}$ will be denoted as $x_{j, k_{1} k_{2}}, x_{j, k_{1} k_{2} k_{3}}$, respectively.

We recall that one should take $x_{j, k}=\delta_{j k}, x_{j, k_{1} k_{2}}=0, x_{j, k_{1} k_{2}, k_{3}}=0$ as initial conditions for $t=0$. In principle, first, second and third variational equations give rise to $4^{2}, 4^{3}$ and $4^{4}$ equations, respectively. But, from one side, these equations have the symmetries of the differential operators $D^{2}$ and $D^{3}$. From the other, due to the special solution chosen and to the simplicity of (38), it is immediate to prove the following lemma.

LEMMA 14. -

(i) The $x_{j, k}$ with $j$ and $k$ of different parity are identically zero.

(ii) For $x_{j, k_{1} k_{2}}$, if the cardinal of the set of indices $k$ which have parity different from the one of $j$, has the parity of $j$, then these elements are identically zero.

(iii) For $x_{j, k_{1} k_{2} k_{3}}$, if the cardinal of the set of indices $k$ which have parity equal to the one of $j$ is even, then these elements are identically zero.

Before starting the analytic computations, some information regarding the expected results is worth inferring. To this end we numerically integrated the required variational equations along paths $\gamma$ as described before. The results show that, when returning to the initial point, the final values of the elements $x_{j, k}$ coincide again with $\delta_{j k}$, the ones of $x_{j, k_{1} k_{2}}$ are again zero and the ones of $x_{j, k_{1} k_{2} k_{3}}$ are also zero with the following exceptions:

$$
x_{2,222}, \quad x_{2,224}, \quad x_{2,244}, \quad x_{2,444}, \quad x_{4,224}, \quad x_{4,244}, \quad x_{4,444} .
$$

Taking a different initial point one can also have $x_{4,222} \neq 0$ after closing the loop. For our purposes it is enough to show that some of the final elements $x_{j, k_{1} k_{2} k_{3}}$ is different from 0 . We select the $x_{2,222}$, whose numerically computed value is $\approx 90.477868423386 \mathrm{i}$.

Let $a_{31}=-1-2 x_{1}, a_{42}=-1-x_{1}$ the only non-zero and non-trivial elements in $D X$ along $\Gamma$. To obtain $x_{2,222}$ we only need to integrate the following systems

$$
\begin{aligned}
& \left(\begin{array}{l}
\dot{x}_{2,2} \\
\dot{x}_{4,2}
\end{array}\right)=\left(\begin{array}{cc}
0 & 1 \\
a_{42} & 0
\end{array}\right)\left(\begin{array}{l}
x_{2,2} \\
x_{4,2}
\end{array}\right), \\
& \left(\begin{array}{c}
\dot{x}_{1,22} \\
\dot{x}_{3,22}
\end{array}\right)=\left(\begin{array}{cc}
0 & 1 \\
a_{31} & 0
\end{array}\right)\left(\begin{array}{l}
x_{1,22} \\
x_{3,22}
\end{array}\right)+\left(\begin{array}{c}
0 \\
-x_{2,2}^{2}
\end{array}\right)
\end{aligned}
$$

and

$$
\left(\begin{array}{c}
\dot{x}_{2,222} \\
\dot{x}_{4,222}
\end{array}\right)=\left(\begin{array}{cc}
0 & 1 \\
a_{42} & 0
\end{array}\right)\left(\begin{array}{c}
x_{2,222} \\
x_{4,222}
\end{array}\right)+\left(\begin{array}{c}
0 \\
-3 x_{2,2} x_{1,22}
\end{array}\right) .
$$

It is clear that to integrate (40) and (41) we need to solve the first-order variational equations, both tangential (in the $\left(x_{1}, x_{3}\right)$ variables) and normal (in $\left(x_{2}, x_{4}\right)$ ), which are uncoupled. The solutions can be written explicitly. To shorten the notation we introduce $c:=\cosh (t / 2)$ and 
$s:=\sinh (t / 2)$. Then

$$
\begin{aligned}
x_{1,1} & =-\frac{15 t s}{16 c^{3}}+\frac{15}{8 c^{2}}-\frac{5}{8}-\frac{c^{2}}{4}, & x_{1,3} & =-\frac{4}{3} y_{1}, \\
x_{3,1} & =-\frac{15 t\left(3-2 c^{2}\right)}{32 c^{4}}-\frac{45 s}{16 c^{3}}-\frac{s c}{4}, & x_{3,3} & =\frac{4}{3}\left(x_{1}+x_{1}^{2}\right), \\
x_{2,2} & =2 x_{1}, & x_{2,4} & =\frac{t x_{1}}{2}+\frac{3 s}{2 c}, \\
x_{4,2} & =2 y_{1}, & x_{4,4} & =\frac{x_{1}}{2}+\frac{t y_{1}}{2}+\frac{3}{4 c^{2}} .
\end{aligned}
$$

Furthermore

$$
x_{1,22}=x_{1,1}\left(\frac{2}{8}-\frac{16}{9} x_{1}^{3}\right)+x_{1,3} K(t)
$$

where

$$
K(t)=t\left(-\frac{45}{16 c^{6}}+\frac{45}{8 c^{4}}-\frac{15}{4 c^{2}}\right)+s\left(-\frac{45}{8 c^{5}}+\frac{15}{2 c^{3}}-\frac{3}{c}+c\right) .
$$

We remark that one of the columns of the fundamental matrix of the normal variational equations coincides (except by a factor of 2) with (39). This is true for any $h$ because $\left(x_{1}, y_{1}\right)$ are solutions of the first equation in (40).

Having (42) and (43) we are ready to solve (41). As the homogeneous part coincides with the first order normal variational equation, the solution, after closing the loop, is given by

$$
\left(\begin{array}{l}
x_{2,222} \\
x_{4,222}
\end{array}\right)=\left(\begin{array}{ll}
x_{2,2} & x_{2,4} \\
x_{4,2} & x_{4,4}
\end{array}\right) \int_{\gamma}\left(\begin{array}{r}
-x_{2,4} R \\
x_{2,2} R
\end{array}\right), \quad \text { where } R(t)=-3 x_{2,2} x_{1,22} .
$$

It is readily checked that the residues inside the integral are $72 / 5$ and 0 , respectively. Hence, the final value of $x_{2,222}$ after the loop is $\frac{72}{5} 2 \pi \mathrm{i}$, which coincides with the value given above in all the digits shown. This computation also explains why the final value of $x_{4,222}$ is zero.

We are now ready to prove the desired non-integrability of (38). We remark that in all the computations of order less than three no residue appears.

PROPOSITION 16. - The system (38) in non-integrable in a vicinity of the solution given by (39).

Proof. - One can take a solution on the invariant plane $x_{2}=y_{2}=0$ as $\Gamma$ and an energy level $h<h^{*}$ close to $h^{*}$. Then the solution is given by elliptic functions with a parallelogram of periods and a double pole. When $h \rightarrow h^{*}$ the periods tend to $\pi \mathrm{i}$ and $\infty$. Take paths $\gamma_{1}$ and $\gamma_{2}$ along the generators. To compute the commutator it is enough to carry out the integration along a path $\gamma$ of index 1 around the pole.

Since all the solutions are obtained by quadratures, we have $\left(G_{k}\right)^{0}=G_{k}$ for all $k$. Hence, commutativity of $\left(G_{k}\right)^{0}$ implies commutativity at the ( $k$-th order) monodromy level.

The integrals along $\gamma$ are continuous as functions of $h$. As for $h=h^{*}$ there are integrals different from zero, the same happens for nearby values $h<h^{*}$. This implies $\left(G_{3}\right)^{0}$ noncommutative.

$4^{\text {e }}$ SÉRIE - TOME $40-2007-\mathrm{N}^{\circ} 6$ 


\section{REFERENCES}

[1] Abraham R., Marsden J.E., Foundations of Mechanics, second ed., Benjamin, 1978.

[2] Adler M., VAn Moerbeke P., VAnhaecke P., Algebraic Integrability, Painlevé Geometry and Lie Algebras, Springer, Berlin, 2004.

[3] Almeida A., López-CAstillo A., Stuchi T., Non-integrability proof of the frozen planetary atom configuration, J. Phys. A: Math. Gen. 36 (2003) 4805-4814.

[4] Almeida A., Stuchi T.J., The integrability of the anisotropic Stormer problem, Physica D 189 (2004) 219-233.

[5] Arribas M., Elipe A., Non Integrability of the motion of a particle around a massive straight segment, Phys. Lett. A 281 (2001) 142-148.

[6] Arribas M., Elipe A., Riaguas A., Non-integrability of anisotropic quasi-homogeneous Hamiltonian systems, Mech. Res. Commun. 30 (2003) 209-216.

[7] ARtin M., On the solutions of analytic equations, Invent. Math. 5 (1968) 277-291.

[8] AтіYAн M.F., MacDonald I.G., Introduction to Commutative Algebra, Addison-Wesley, London, 1969.

[9] Audin M., Intégrabilité et non-intégrabilité de systèmes hamiltoniens (d'après S. Ziglin, J. MoralesRuiz, J.-P. Ramis, ... ), Sém. Bourbaki (2000/01), Exp. nº 884, Astérisque 282 (2002) 113-135.

[10] AUdin M., Les systèmes hamiltoniens et leur intégrabilité, Cours Spécialisés, Collection SMF, vol. 8, Société Mathématique de France, Marseille, 2001.

[11] AUdin M., Integrability of Hamiltonian systems, Bulletin E.M.S. (December 2003) 9-12.

[12] Audin M., Exemples de hamiltoniens non intégrables en mécanique analytique réelle, Ann. Fac. Sci. Toulouse Math. 12 (2003) 1-23.

[13] AUdin M., La réduction symplectique appliquée à la non-intégrabilité du problème du satellite, Ann. Fac. Sci. Toulouse Math. 12 (2003) 25-46.

[14] BOUCHER D., Sur la non-intégrabilité du problème plan des trois corps de masses égales à un le long de la solution de Lagrange, C. R. Acad. Sci. Paris, Série I 331 (2000) 391-394.

[15] Boucher D., Sur les équations différentielles linéaires paramétrées, une application aux systèmes hamiltoniens, Thèse Univ. de Limoges, octobre 2000.

[16] Boucher D., Non complete integrability of a satellite in circular orbit, Portugaliae Mathematica (N.S.) 63 (2006) 69-89.

[17] Boucher D., WeIL J.-A., Application of the J.-J. Morales and J.-P. Ramis theorem to test the noncomplete integrability of the planar three-body problem, in: Fauvet F., Mitschi C. (Eds.), From Combinatorics to Dynamical Systems. Journées de calcul formel en l'honneur de Jean Thomann, March 22-23, 2002, in: IRMA Lectures in Mathematics and Theoretical Physics, vol. 3, IRMA, Strasbourg, 2003.

[18] BourbaKi N., Algèbre commutative, chapitres 1 à 4, Springer-Verlag, 2006.

[19] Bryant R.L., Chern S.S., Gardner R.B., Goldschmidt H.L., Griffiths P.A., Exterior Differential Systems, Springer-Verlag, Berlin, 1991.

[20] Casale G., Sur le groupoïde de Galois d'un feuilletage, Thèse, Toulouse 2004.

[21] Churchill R.C., Galoisian Obstructions to the Integrability of Hamiltonian Systems, The Kolchin Seminar in Differential Algebra, City College of New York, May, 1998.

[22] Churchill R.C., Differential algebraic techniques in Hamiltonian mechanics, in: Guo L., , Keigher W.F., Cassidy P.J., Sit W.Y. (Eds.), Differential Algebra and Related Topics, World Scientific Publ., 2002, pp. 219-255.

[23] Deligne P., Catégories tannakiennes, in: Grothendieck festschrift, vol. 2, in: Progress in Mathematics, vol. 87, Birkhäuser, 1990, pp. 111-196.

[24] Doubrovine B., Novikov S., Fomenko A., Géométrie Contemporaine, Méthodes et Applications, Deuxième partie, Géométrie et Topologie des Variétés, Éditions MIR, Moscou, 1979 (French translation 1982).

[25] DRACH J., Essai sur une théorie générale de l'intégration et sur la classification des transcendantes, Ann. Sci. École Normale Sup., Sér. 315 (1898) 243-384.

[26] Drach J., Sur le problème logique de l'intégration des équations différentielles, Ann. Fac. Sci. Univ. Toulouse, Sér. 210 (1908) 393-472. 
[27] Ferrer S., MondéJAR F., On the non-integrability of the Zeemann-Stark Hamiltonian system, Comm. Math. Phys. 208 (1999) 55-63.

[28] Ferrer S., MondÉJAR F., Non-integrability of the 3-D Hydrogen atom under motional Stark effect or circularly polarized microwave combined with magnetic fields, Phys. Lett. A 264 (1999) 74-83.

[29] Ferrer S., MondéJAR F., On the non-integrability of the generalized van der Waals Hamiltonian, J. Math. Phys. 41 (2000) 5445-5452.

[30] Fontich E., SIMÓ C., Invariant manifolds for near identity differentiable maps and splitting of separatrices, Erg. Th. \& Dyn. Systems 10 (1990) 319-346.

[31] Fontich E., Simó C., The splitting of separatrices for analytic diffeomorphisms, Erg. Th. \& Dyn. Systems 10 (1990) 295-318.

[32] Grothendieck A., Techniques de construction en géométrie analytique VII, Séminaire Henri Cartan, 13-ème année 1960/61, vol. 14, W.A. Benjamin, 1967.

[33] HÉnOn M., HeIles C., The applicability of the third integral of motion: Some numerical experiments, Astronom. J. 69 (1964) 73-79.

[34] IrigoYen M., Simó C., Non-integrability of the $J_{2}$ problem, Celest. Mech. 55 (1993) 281-287.

[35] Iто H., Non-integrability of the Hénon-Heiles system and a theorem of Ziglin, Koday Math. J. 8 (1985) 129-138.

[36] Iто H., On the holonomy group associated with analytic continuations of solutions for integrable systems, Boletim da Soc. Brasil. de Matemática 21 (1990) 95-120.

[37] Juillard Tosel E., Non-integrabilité algébrique et méromorphe de problèmes de N corps, Thèse Univ. Paris VI, janvier 1999.

[38] Juillard Tosel E., Meromorphic parametric non-integrability; the inverse square potential, Arch. Rat. Mech. Anal. 152 (2000) 187-207.

[39] Kolar I., Michor P., SlovaK J., Natural Operations in Differential Geometry, Springer-Verlag, 1991.

[40] Levine H.I., Singularities of differentiable mappings, in: Proceedings of Liverpool Singularities Symposium I, in: Lecture Notes in Math., vol. 192, 1971.

[41] Llibre J., Simó C., Homoclinic phenomena in the three-body problem, J. Differential Equations 37 (1980) 444-465.

[42] LlibRe J., Simó C., Oscillatory solutions in the planar restricted three-body problem, Math. Ann. 248 (1980) 153-184.

[43] Llibre J., Martínez R., Simó C., Transversality of the invariant manifolds associated to the Lyapunov family of periodic orbits near $L_{2}$ in the Restricted Three Body Problem, J. Differential Equations 58 (1985) 104-156.

[44] MACIEJEWSKI A.J., Non-integrability of certain Hamiltonian systems, applications of the MoralesRamis differential Galois extension of Ziglin theory, Banach Center Publications 58 (2002) 139150.

[45] Maciejewski A.J., PrZybylska M., Non-integrability of restricted two-body problems in constant curvature spaces, Regul. Chaotic Dyn. 8 (4) (2003) 413-430.

[46] Maciejewski A.J., Przybylska M., Non-integrability of the problem of a rigid satellite in gravitational and magnetic fields, Celest. Mech. Dynam. Astron. 87 (2003) 317-351.

[47] Maciejewski A.J., Przybylska M., Non-integrability of the Suslov problem, J. Math. Phys. 45 (2004) 1065-1078.

[48] Maciejewski A.J., Przybylska M., Non-integrability of the generalised two fixed centres problem, Celest. Mech. Dynam. Astron. 89 (2004) 145-164.

[49] Maciejewski A.J., PrZYbylska M., All meromorphically integrable 2D Hamiltonian systems with homogeneous potential of degree 3, Phys. Lett. A 327 (2004) 461-473.

[50] Maciejewski A.J., Przybylska M., Differential Galois approach to the non-integrability of the heavy top problem, Ann. Fac. Sci. Toulouse Sér. 614 (2005) 123-160.

[51] Maciejewski A.J., Przyb Ylska M., Stachowiak T., Non integrability of Gross-Neveu systems, Physica D 201 (2005) 249-267.

[52] Maciejewski A.J., Przybylska M., Weil J.-A., Non-integrability of the generalized springpendulum problem, J. Phys. A: Math. Gen. 37 (2004) 2579-2597.

$4^{\mathrm{e}}$ SÉRIE - TOME $40-2007-\mathrm{N}^{\circ} 6$ 
[53] Maciejewski A.J., Strelcyn J.M., Szydlowski M., Non-integrability of Bianchi VIII Hamiltonian system, J. Math. Phys. 42 (2001) 1728-1743.

[54] Maciejewski A.J., Szydlowski M., Towards a description of complexity of the simplest cosmological systems, J. Phys. A: Math. Gen. 33 (2000) 9241-9254.

[55] Maciejewski A.J., Szydlowski M., Integrability and non-integrability of planar Hamiltonian systems of cosmological origin, J. Nonlinear Math. Phys. 8 (2001) 200-206.

[56] Malgrange B., Le groupoïde de Galois d'un feuilletage, L'enseignement mathématique 38 (2) (2001) 465-501.

[57] Malgrange B., On the non linear Galois theory, Chinese Ann. Math. Ser. B 23 (2) (2002) 219-226.

[58] Martinet J., Singularités des fonctions et applications différentiables, Pontificia Universidade Católica do Rio de Janeiro (PUC), 1974.

[59] Morales-Ruiz J.J., Differential Galois Theory and Non-Integrability of Hamiltonian Systems, Progress in Mathematics, vol. 179, Birkhäuser, 1999.

[60] Morales-Ruiz J.J., Kovalevskaya, Liapounov, Painlevé, Ziglin and the differential Galois theory, Regular Chaotic Dynam. 5 (2000) 251-272.

[61] Morales-Ruiz J.J., Peris J.M., On a Galoisian approach to the splitting of separatrices, Ann. Fac. Sci. Toulouse Math. 8 (1999) 125-141.

[62] Morales-Ruiz J.J., RAmis J.P., Galoisian obstructions to integrability of Hamiltonian systems, Methods Appl. Anal. 8 (2001) 33-96.

[63] Morales-Ruiz J.J., RAmis J.P., Galoisian obstructions to integrability of Hamiltonian systems II, Methods Appl. Anal. 8 (2001) 97-112.

[64] Morales-Ruiz J.J., RAmis J.P., A note on the non-integrability of some Hamiltonian systems with a homogeneous potential, Methods Appl. Anal. 8 (2001) 113-120.

[65] MoRAlES-RUIZ J.J., SiMó C., Non-integrability criteria for Hamiltonians in the case of Lamé normal variational equations, J. Differential Equations 129 (1996) 111-135.

[66] Morales-Ruiz J.J., Simó C., Simon S., Algebraic proof of the non-integrability of Hill's problem, Ergod. Th. \& Dynam. Sys. 25 (2005) 1237-1256.

[67] Moser J., Stable and Random Motions in Dynamical Systems, Annals of Mathematics Studies, Princeton Univ. Press, 1973.

[68] NAKAGAWA K., Direct construction of polynomial first integrals for Hamiltonian systems with a two-dimensional homogeneous polynomial potential, Dep. of Astronomical Science, The Graduate University for Advanced Study and the National Astronomical Observatory of Japan, Ph.D. Thesis, 2002.

[69] NAKAGAWA K., Yoshida H., A necessary condition for the integrability of homogeneous Hamiltonian systems with two degrees of freedom, J. Phys. A: Math. Gen. 34 (2001) 2137-2148.

[70] NeIshtadT A.I., The separation of motions in systems with rapidly rotating phase, Prikladnaja Matematika i Mekhanika 48 (1984) 133-139.

[71] Poincaré H., Les Méthodes Nouvelles de la Mécanique Céleste, vol. I, Gauthiers-Villars, Paris, 1892.

[72] SAEnZ A.W., Nonintegrability of the Dragt-Finn model of magnetic confinement: A Galoisian-group approach, Physica D 144 (2000) 37-43.

[73] Serre J-P., Géométrie algébrique et géométrie analytique, Ann. Inst. Fourier Grenoble (1956) 1-42.

[74] Serre J-P., Espaces fibrés algébriques, in: Séminaire Claude Chavalley, 1958, tome 3, exp. nº 1, pp. $1-37$.

[75] SIMÓ C., Analytical and numerical computation of invariant manifolds, in: Benest D., Froeschlé C. (Eds.), Modern Methods in Celestial Mechanics, Editions Frontières, 1990, pp. 285-330. Also available at http://www.maia.ub.es/dsg/.

[76] Simó C., Measuring the lack of integrability in the $J_{2}$ problem, in: Roy A.E. (Ed.), Predictability, Stability and Chaos in the N-Body Dynamical Systems, Plenum Press, 1991, pp. 305-309.

[77] TERNG C.L., Natural vector bundles and natural differential operators, Amer. J. Math. 100 (1978) $775-828$.

[78] Tsygvintsev A., Sur l'absence d'une intégrale première méromorphe supplémentaire dans le problème plan des trois corps, C. R. Acad. Sci. Paris Série I 333 (2001) 241-244. 
[79] UMEmURa H., Differential Galois theory of infinite dimension, Nagoya Math. J. 144 (1996) 59-135.

[80] VAn Der Put M., Singer M., Galois Theory of Linear Differential Equations, Springer, Berlin, 2003.

[81] Vigo-Aguilar M.I., No integrabilidad del problema del satélite, Ph.D. Thesis, Departamento de Análisis Matemático y Matemática Aplicada, Universidad de Alicante, 1999.

[82] YAGASAKI K., Galoisian obstructions to integrability and Melnikov criteria for chaos in two-degreeof-freedom Hamiltonian systems with saddle-centers, Nonlinearity 16 (2003) 2003-2013.

[83] YAGASAKI K., Non-integrability of an infinity-degree-of-freedom model for unforced and undamped straight beams, J. Appl. Mech. 70 (2003) 732-738.

[84] YoshidA H., A new necessary condition for the integrability of Hamiltonian systems with a two dimensional homogeneous potential, Physica D 128 (1999) 53-69.

[85] YoshidA H., Justification of Painlevé Analysis for Hamiltonian systems by differential Galois theory, Physica A 228 (2000) 424-430.

[86] ZIGLIN S.L., Branching of solutions and non-existence of first integrals in Hamiltonian mechanics I, Funct. Anal. Appl. 16 (1982) 181-189.

(Manuscrit reçu le 8 septembre 2005; accepté, après révision, le 21 septembre 2007.)

Juan J. MORALES-RUIZ

Departament de Matemàtica Aplicada II,

Universitat Politècnica de Catalunya,

Edifici Omega,

Campus Nord,

c/ Jordi Girona, 1-3,

E-08034 Barcelona, Spain

E-mail: Juan.Morales-Ruiz@upc.edu

Jean-Pierre RAMIS

Académie des Sciences,

Institut Universitaire de France and

Laboratoire Émile Picard,

Université Paul Sabatier,

118, route de Narbonne,

31062 Toulouse Cedex, France

E-mail: ramis@ picard.ups-tlse.fr

Carles Simó

Departament de Matemàtica Aplicada i Anàlisi, Universitat de Barcelona,

Gran Via 585,

E-08007 Barcelona, Spain

E-mail: carles@maia.ub.es

$4^{\mathrm{e}}$ SÉRIE - TOME $40-2007-\mathrm{N}^{\circ} 6$ 